\title{
DAPK1 loss triggers tumor invasion in colorectal tumor cells
}

\author{
Sara Steinmann $\mathbb{1}^{1,2,3}$, Philipp Kunze ${ }^{1,2}$, Chuanpit Hampel ${ }^{1,2}$, Markus Eckstein², Jesper Bertram Bramsen ${ }^{4}$, \\ Julienne K. Muenzner ${ }^{1,2}$, Birgitta Carlé, Benardina Ndreshkjana ${ }^{1,2}$, Stephan Kemenes ${ }^{1,2}$, Pierluigi Gasparini 6 , \\ Oliver Friedrich ${ }^{5}$, Claus Andersen $\mathbb{1}^{4}$, Carol Geppert ${ }^{2}$, Shengbao Wang ${ }^{7}$, Ilker Eyupoglu' ${ }^{7}$, Tobias Bäuerle ${ }^{8}$, \\ Arndt Hartmann ${ }^{2}$ and Regine Schneider-Stock ${ }^{1,2}$
}

\begin{abstract}
Colorectal cancer (CRC) is one of the leading cancer-related causes of death worldwide. Despite the improvement of surgical and chemotherapeutic treatments, as of yet, the disease has not been overcome due to metastasis to distant organs. Hence, it is of great relevance to understand the mechanisms responsible for metastasis initiation and progression and to identify novel metastatic markers for a higher chance of preventing the metastatic disease. The Death-associated protein kinase 1 (DAPK1), recently, has been shown to be a potential candidate for regulating metastasis in CRC. Hence, the aim of the study was to investigate the impact of DAPK1 protein on CRC aggressiveness. Using CRISPR/Cas9 technology, we generated DAPK1-deficient HCT116 monoclonal cell lines and characterized their knockout phenotype in vitro and in vivo. We show that loss of DAPK1 implemented changes in growth pattern and enhanced tumor budding in vivo in the chorioallantoic membrane (CAM) model. Further, we observed more tumor cell dissemination into chicken embryo organs and increased invasion capacity using rat brain 3D in vitro model. The novel identified DAPK1-loss gene expression signature showed a stroma typical pattern and was associated with a gained ability for remodeling the extracellular matrix. Finally, we suggest the DAPK1-ERK1 signaling axis being involved in metastatic progression of CRC. Our results highlight DAPK1 as an anti-metastatic player in CRC and suggest DAPK1 as a potential predictive biomarker for this cancer type.
\end{abstract}

\section{Introduction}

Colorectal cancer $(\mathrm{CRC})$ is the fourth most frequently diagnosed malignancy worldwide. Approximately $8.5 \%$ of patients with CRC die from their cancer ${ }^{1}$. Development of metastasis predominantly in liver and lung is the fatal leading cause of death and a serious obstacle of curing CRC. Recently, it has been shown that Death-associated protein kinase 1 (DAPK1) might be a potential candidate

\footnotetext{
Correspondence: Regine Schneider-Stock (regine.schneider-stock@ukerlangen.de)

${ }^{1}$ Experimental Tumor Pathology, University Hospital Erlangen, FriedrichAlexander University Erlangen-Nuremberg, Universitaetsstrasse 22, 91054 Erlangen, Germany

${ }^{2}$ Institute of Pathology, University Hospital Erlangen, Friedrich-Alexander University Erlangen-Nuremberg, Krankenhausstr. 8-10, 91054 Erlangen, Germany Full list of author information is available at the end of the article.

Edited by M. Herold
}

regulating metastasis in $\mathrm{CRC}^{2-4}$. DAPK1 as an actin filament-associated calcium/calmodulin-regulated, stressresponsive serine/threonine kinase was reported to be an extremely pleiotropic molecule due to its unique multidomain structure ${ }^{5-9}$. Chen et al. $^{3}$ have postulated that three main action modes for metastasis suppression by DAPK1 might exist: the increase in susceptibility of tumor cells to apoptotic signals, inhibitory role on integrinmediated cell adhesion and migration and modulation of tumor microenvironment. Several reports have shown that primary tumors of patients with metastases (pM1) show decreased DAPK1 protein expression in comparison to primary tumors without metastases (pM0) ${ }^{3,4}$. Recently we have reported that the loss of anti-migratory function of DAPK1 could be one of the reasons for tumor cell dissemination ${ }^{4}$. In this regard it was not surprising that

\section{(c) The Author(s) 2019}

(c) (i) Open Access This article is licensed under a Creative Commons Attribution 4.0 International License, which permits use, sharing, adaptation, distribution and reproduction cc) in any medium or format, as long as you give appropriate credit to the original author(s) and the source, provide a link to the Creative Commons license, and indicate if changes were made. The images or other third party material in this article are included in the article's Creative Commons license, unless indicated otherwise in a credit line to the material. If material is not included in the article's Creative Commons license and your intended use is not permitted by statutory regulation or exceeds the permitted use, you will need to obtain permission directly from the copyright holder. To view a copy of this license, visit http://creativecommons.org/licenses/by/4.0/. 
DAPK1 was nearly lost in tumor buds at the invasion front of CRC. Tumor buds are defined as single cells or small clusters of up to four cells ${ }^{4}$ and their evaluation at the stroma border of colorectal tumors is supposed to be indicative for tumor aggressiveness ${ }^{4}$. Moreover, tumor budding seems to be an independent and a reliable predictor of local lymph node metastasis, tumor recurrence and survival ${ }^{10}$. Since DAPK1 is lost in this aggressive cell subpopulation at the tumor invasion front we suggest a tumor suppressor role for DAPK1. Interestingly, DAPK1 was found to be involved in the cross talk of tumor cells with macrophages of the stroma environment ${ }^{11}$. At the onset of cancer, loss of DAPK1 may provide selective advantage for hyperproliferative tumor cells of evading the p53-dependent apoptotic checkpoint during transformation $^{12}$. Indeed, DAPK1 loss has been shown in a high number of T1 colorectal tumors ${ }^{13}$. At later stages of tumorigenesis and during metastasis, tumor cells seem to benefit from loss of DAPK1 resulting in reduced sensitivity to detachment from extracellular matrix $(\mathrm{ECM})^{14}$. So far, the molecular mechanisms behind metastasis in $\mathrm{CRC}$ and especially the role of DAPK1 in this process remain little understood.

To mirror-image DAPK1 loss in tumor buds and to shed more light into the role of DAPK1 in CRC aggressiveness we designed unique CRISPR/Cas9 DAPK1 knockout (ko) cell lines and characterized the knockout phenotype in vitro and in vivo. Although we observed a definite variation in functional readouts for the different clones, we were able to extract a uniform DAPK1-stroma specific gene expression pattern. In the chorioallantoic membrane (CAM) model, we showed an increased tumor budding and metastatic potential when DAPK1 was lost in tumor cells supporting its role as a tumor suppressor. We propose the DAPK1-ERK axis to be involved in metastasis suppression. Loss of adhesion molecule ICAM1, gain in TACSTD2 expression, and increased tumor cell-ECM interaction under DAPK1 loss seem to be central events in this process.

\section{Material and methods}

\section{Cell lines, cell culture, and ERK1/2 inhibition}

The human colorectal tumor cell lines HCT116, DLD1, SW480, SW620, SW837, and HT29 were obtained from ATCC. HCT116 cells line was used as in vitro model due to wildtype Tp53 status compared to other CRC cell lines (Supplementary Table 1). HCT116 and HCT116-derived monoclonal DAPK1 ko clones $7 / 6,10 / 8$ and 21/9 were maintained in RPMI 1640 medium supplemented with $10 \%(\mathrm{v} / \mathrm{v})$ fetal bovine serum (FBS), 1\% penicillin (100 U/ $\mathrm{ml})$ and streptomycin $(100 \mu \mathrm{g} / \mathrm{ml})$ (all from PAN Biotech, Aidenbach, Germany) and cultured in a humidified atmosphere of $5 \% \mathrm{CO}_{2}$ at $37^{\circ} \mathrm{C}$. For ERK1/2 inactivation, cells were treated with 10 and $20 \mu \mathrm{M}$ FR180204
(Selleckchem, Munich, Germany) for $48 \mathrm{~h}$. All cell lines were genotyped using Multiplex Cell Authentication by Multiplexion (Heidelberg, Germany) as described recently ${ }^{15}$. Mycoplasma-free status has been verified for all cell lines.

\section{Establishment of DAPK1 ko cell lines}

HCT116 cells were genome-edited using the CRISPR/ Cas9 technology ${ }^{16}$. CRISPR/Cas9 vector pX330 was a gift from Feng Zhang (plasmid \#42230, Addgene, Teddington, UK). The antibiotic selection vector pBABE-puro (Addgene plasmid \#1764) was kindly provided by Professor Jeffrey Parvin (OSU, Columbus OH, USA). For CRISPR/Cas9-mediated gene ko, single guide RNAs (sgRNA) targeting exon 3 of human DAPK1 (NM_004938; ENSG00000196730; sgRNA1: nt 611-629, sgRNA2: nt 615-634; kinase domain) were designed using a common CRISPR design tool (https://benchling.com/ academic; Supplementary Fig. 1a). After annealing, the 20 nt targeting sgRNA (Supplementary Fig. 1b) were introduced into pX330 at its BbsI site. For transient transfection, $0.3 \times 10^{6}$ cells per 6 well were seeded and cultured for approximately $24 \mathrm{~h}$ until $70-80 \%$ of confluency. $1.25 \mu \mathrm{g}$ of pX330-DAPK1-sgRNA1 or pX330-DAPK1sgRNA2 and $1.25 \mu \mathrm{g}$ of pBABE-puro (plasmid \#1764, Addgene, Teddington, UK $)^{17}$ for antibiotic selection were transiently co-transfected into adherent HCT116 cells using Lipofectamine 2000 (Life Technologies/Thermo Fisher Scientific, Waltham, MA, USA) according to the manufacturer's instructions. After $24 \mathrm{~h}$ transfected cells were maintained in culture medium containing $1.5 \mathrm{mg} / \mathrm{ml}$ puromycin for 19 days for positive selection. For isolation of monoclonal cell populations, surviving cells were harvested and seeded as limiting dilution $(100 \mu \mathrm{l}$ of a $4-5$ cells $/ \mathrm{ml}$ solution per 96 well). Single-cell colonies were expanded for DNA- and protein extraction and cryopreservation. Each clone was genotyped by Sanger sequencing (Seqlab, Germany) of PCR-amplified gDNA (sense: $5^{\prime}$ TCA ATC CCT CGT TTT TCA GG -3', anti-sense: $5^{\prime}$ CCA ATT CCT GAT CCC TCT CTC $-3^{\prime}$ ) using the forward primer 5'- CCA CAT CCT CAC TCA AAT CCT -3'.

\section{Nuclear/cytoplasmic fractionation of proteins}

Sub-cellular fractions of the HCT116, HCT 7/6, and HCT 21/9 cells were prepared using REAP cell fractionation method ${ }^{18}$. Briefly, cell pellets were resuspended in $500 \mu \mathrm{l}$ of ice-cold $0.1 \%$ NP40 (Calbiochem, CA, USA) in PBS, triturated five times using a p1000 micropipette and centrifuged for $10 \mathrm{~s}$ in $1.5 \mathrm{ml}$ micro-centrifuge tubes. The supernatants were transferred to the new tubes and kept on ice (this is the cytoplasmic fraction). The pellets were washed with $1 \mathrm{ml}$ of ice-cold 0.1\% NP40-PBS lysis buffer, centrifuged for $10 \mathrm{~s}$, and the supernatants were discarded. The remaining pellet was dissolved in $100 \mu \mathrm{l} 0.1 \%$ 
NP40-PBS lysis buffer (this is the nuclear fraction). All lysates were analyzed by Western Bloting.

\section{Western Blotting analysis}

Western Blotting was performed as previously described $^{4}$. Briefly whole cell lysates were prepared in urea lysis buffer (4 M urea, 0.5\% SDS, $62.5 \mathrm{mM}$ Tris, pH 6.8) supplemented with $1 \%$ Protease inhibitor cocktail (Merck Millipore, Darmstadt, Germany) and $1 \mathrm{mM}$ phenylmethylsulfonylfluorid (Roth, Karlsruhe, Germany). Sodium dodecyl sulfate polyacrylamide (PAA) Gel Electrophoresis (SDS-PAGE; 7.5-12\% of PAA) was performed with $30-60 \mu \mathrm{g}$ protein per sample and proteins were transferred onto nitrocellulose membranes (Whatman, Little Chalfont, UK) overnight. After blocking membranes were incubated with primary antibodies at $4{ }^{\circ} \mathrm{C}$ overnight and then horseradish-peroxidase (HRP)-conjugated secondary antibodies anti-mouse and anti-rabbit (1:10 000; Thermo Fisher Scientific, Waltham, MA, USA) were added for $1 \mathrm{~h}$ at RT. Chemiluminescence images were captured using the Gene Gnome chemiluminescence developer (Syngene, Bangalore, India). The primary antibodies were: anti-Cofilin (1:1000, sc-33779), -phosphoCofilin $^{\text {Ser3 }}$ (1:500, sc-12912-R; both from Santa Cruz, Dallas, TX, USA), -DAPK1 (1:150, 610291; BD Biosciences, Heidelberg, Germany), -DAPK2 (1:250, PA141305; Life Technologies/Thermo Fisher Scientific, Waltham, MA, USA), -DRAK1 (1:500, PA5-21849), -DRAK2 (1:500, PA1-41308; both from Thermo Fisher Scientific, Waltham, MA, USA), hSpCas9 (1:1000, C152002203; Diagenode, Seraing, Belgium), CD133 (1:250, 130-092-395; Miltenyi Biotec GmbH, Bergisch Gladbach, Germany), Lamin A + C (1:4000, AB108922); Abcam, Berlin Germany) -ERK1/2 (1:1000, 9102), pERK1/ 2 (1:1 000, 9101), -ICAM1 (1:250, 4915), -DAPK3 (1:1000, 2928), -CD44 (1:1000, 3570), -Vimentin (1:1000, 5741), -E-Cadherin (1:1000, 3195), p-MLC (1:500, 3671), and -TACSTD2 (1:1 000, 90540); all from Cell Signaling, Frankfurt am Main, Germany), Western Blot bands were quantified by densitometric analysis using ImageJ (National Institutes of Health; Bethesda, MD, USA). HRPconjugated anti-GAPDH (1:75 000, MAB5476; Abnova, Aachen, Germany) served as loading control for protein normalization. Experiments were performed at least two times.

\section{WST-8-based cell proliferation assay}

Proliferation rate was determined using the colorimetric Cell Counting Kit-8 (CCK-8, Dojindo, Munich, Germany) according to the manufacturer's instructions. Briefly, cells $\left(10 \times 10^{4}\right.$ cells/well $)$ were seeded in a 96 well flat-bottom microplate and cultured in $200 \mu \mathrm{l}$ of culture medium at $37^{\circ} \mathrm{C}$ and $5 \% \mathrm{CO}_{2}$ overnight to allow adherence and further cultured for $0,3,6,24$ and $48 \mathrm{~h}$. After given time points, old medium was replaced by $100 \mu$ fresh medium supplemented with 1\% WST-8 reagent and incubated for further $2 \mathrm{~h}$ at $37^{\circ} \mathrm{C}$. Thereafter, $100 \mu \mathrm{l}$ supernatant was transferred into a new 96 well and the absorbance was measured at $450 \mathrm{~nm}$ using the multilabel reader VIC$\mathrm{TOR}^{\mathrm{TM}} \mathrm{X} 3$ (Perkin Elmer, Rodgau, Germany). Results are presented as mean \pm SEM. A value of $P<0.05$ was considered to be statistically significant.

\section{Chicken CAM assay}

Fertilized, specific pathogen-free (SPF) chicken eggs (VALO Biomedia, Osterholz-Scharmbeck, Germany) were maintained at $37^{\circ} \mathrm{C}$ and $80 \%$ constant humidity. On day 8 , a window of $1.5-2.0 \mathrm{~cm}$ diameter was cut in the shell at the more rounded pole of the egg and sealed with tape (Durapore silk tape, $3 \mathrm{M}$ ). The next day, $1.0 \times 10^{6}$ human tumor cells per pellet were embedded in growth factorreduced matrigel (Corning, Wiesbaden, Germany) serving as matrix and were transplanted onto the CAM. The window was sealed again and eggs were incubated for additional 5 days. Tumor growth was monitored over time using a light microscope $(\times 10$, SU 1071 Traveler). Tumors were sampled with the surrounding CAM on day 5 , fixed in $4 \%$ formaldehyde, paraffin-embedded and cut into 3-5 $\mu \mathrm{m}$ sections for immunohistochemical evaluation. For gene expression and protein expression analysis, fresh tumors were cryopreserved in liquid nitrogen and stored at $-80^{\circ} \mathrm{C}$ for long-term storage (44). For optical imaging, tumor cells were stained with CytoPainter Cell Proliferation Staining Reagent-Deep Red Fluorescence (ab176736, Abcam, Cambridge, UK) as described previously ${ }^{19}$.

\section{Immunohistochemistry}

Serial sections $(3-5 \mu \mathrm{m})$ of $4 \%$ formalin-fixed and paraffin-embedded CAM tumors were deparaffinized with xylene and rehydrated in graded alcohol. Validated protocols established for the clinical routine were applied for hematoxylin-eosin (HE), phospho-Histone 3 (pHH3, Cell Signaling, Frankfurt am Main, Germany), panCytokeratin (pan-CK, Thermo Fisher Scientific, Waltham, MA, USA) staining. Anti-ICAM1 and anti-phospho-ERK1/2 ${ }^{\text {Thr202/Tyr204 }}$ (both from Cell Signaling, Frankfurt am Main, Germany) and anti-TACSTD2 (Abcam, Cambridge, UK) stainings were performed as follows: for antigen retrieval, slides were cooked in $1 \mathrm{mM}$ Tris-EDTA buffer (pH 8.5) at $120^{\circ} \mathrm{C}$ for $5 \mathrm{~min}$. After peroxidase blocking (Dako/Agilent, Munich, Germany) for $5 \mathrm{~min}$ at RT, slides were incubated with primary antibodies anti-ICAM1 (1:50) and anti-phospho-ERK1/ $2^{\text {Thr202/Tyr204 }}$ (1:50) for $30 \mathrm{~min}$ and with anti-TACSTD2 (1:2000) overnight at RT. ICAM1 and anti-phosphoERK1/2 $2^{\text {Thr202/Tyr204 }}$ antibody binding was visualized by incubating the sections with the EnVision Detection System (Peroxidase/DAB, Dako) for $30 \mathrm{~min}$ at RT and 
subsequently with DAB substrate (Dako/Agilent, Munich, Germany) for $10 \mathrm{~min}$ at RT. For detection of TACSTD2 antibody binding, the ABC-kit from Vector Laboratieries (Burlingame, CA, USA) was used according to the manufacturer's recommendations. Sections were counterstained with hematoxylin (Merck) for $1 \mathrm{~min}$. Immunohistological stainings were brightfield imaged at a magnification of $\times 20$ with the Olympus BX51 microscope and Olympus XC50 camera (Olympus Corporation, Hamburg, Germany) or were scanned using a Panoramic MIDI system (Camera type: CIS VCC-FC60FR19CL; objective: Plan-Apochromat; magnification: $\times 40$; Camera adapter magnification: $\times 1,3$ DHISTECH, Ludwigshafen, Germany) for digital analysis. Tumor budding ${ }^{10}$ of CAM tumors was determined using pan-CK stained sections and the high-power-field (HPF, $\times 40) \operatorname{method}^{20}$. It was calculated as the average number of buds in 4-10 HPFs per sample. A respective budding score was defined as low-grade with an average of $\leq 1$ and high-grade with an average of $>1$ buds per 4-10 HPFs. Vessel areas of CAM xenografts were determined in scans of HE stained sections. The tumor as well as the intratumoral vessels were annotated manually using the CaseViewer software (3DHISTECH, Ludwigshafen, Germany). The percentage of vessel area was calculated by relating the total area of intratumoral vessels to tumor area and expressed in percent (\%). ICAM1, TACSTD2 and cytoplasmic and nuclear pERK immunoreactive scores (IRS) were determined by multiplying staining intensities $(0-3)$ and the respective percentage of positive cells $(0-100 \%$, no positive cell -0 ; all cells positive -100 ) according to Remmele and Stegner ${ }^{1}$. The product was divided by 10 to achieve an IRS between 0 and 27. The mitotic rate of cells in the CAM micro-tumors was determined using $\mathrm{pHH} 3$ stained sections. Quantification was conducted using the QuPath software (https://qupath.github.io/) semi-automatically analyzing $10 \mathrm{HPFs}(\times 20$ magnification) per section. The mitotic index per section is expressed as the mean number of positive mitotic figures in $\%$ of $10 \mathrm{HPFs}$.

\section{Ex ovo optical imaging}

After performing the CAM assay using HCT116 and clone 21/9 cells, chicken embryos were sacrificed by decapitation on day 6 of tumor growth and were placed in an in vivo optical whole body imaging system (IVIS Spectrum CT, Perkin Elmer Rodgau, Germany). On the camera of the imaging system, images of the whole embryo were taken and overlayed with optical signals of deep red fluorescence labeled cells (CytoPainter Cell Proliferation Staining Reagent, Abcam, Cambridge, UK) which were acquired with the following parameters: Epiillumination using an excitation filter for $605 \mathrm{~nm}$ and an emission filter for $660 \mathrm{~nm}$, exposure time of $2 \mathrm{~s}$ and optical fields of view (FOV B) of $6.6 \mathrm{~cm}$. After background correction (embryos with unstained tumor cells), the average radiant efficiency within the embryos was determined by selecting a rectangular ROI that covered the entire embryo. Embryos without tumor cell grafts served as baseline control.

\section{Organotypic brain slice culture}

The brain slice culture system using genetically identical slices of 6-day-old rat (Wistar strain, Charles River) brains were utilized in order to study colorectal carcinoma cell invasion. HCT116, clone $7 / 6$, clone $10 / 8$ and clone $21 / 9$ cells were labeled with a green fluorescent vital dye (Abcam, Cambridge, UK) for tracking invading cells and subsequently transplanted onto the brain slices $\left(3.0 \times 10^{5}\right.$ cells per brain slice). For each cell line, brain precision-cut tissue slices (PCTS, 3 slices per 6 well) of 5 different rats were used. Slices of a sixth rat without tumor cell transplants served as negative control. Propidium iodide (PI) staining of all brain slices served as control for equal loss of vitality of the brain tissue (data not shown). The integrated fluorescence intensities of live invading tumor cells and PI signals were imaged ( $\times 2.0$ magnification $)$ and quantified after 1 and 3 days of tumor growth under a fluorescence microscope (Olympus IX71, Olympus, Hamburg, Germany). Data are reported as mean fluorescent intensities per brain slice.

\section{D-tumor spheroid-based migration assay}

For spheroid formation, HCT116 cells and clone 7/6, clone $10 / 8$ and clone $21 / 9$ cells $\left(2.0 \times 10^{3}\right.$ cells per 96 well $)$ were seeded into a 96 well round-bottom plate. After 3 days, preformed multicellular spheroids were transferred into an uncoated 96-well flat-bottomed plate (one spheroid per well). Tumor cell migration/dissemination was monitored for 48 and $96 \mathrm{~h}$ and imaged using a light microscopy (Leica Dmi1 light microscope, $\times 10$ HI Plan I objective, Leica, Munich, Germany). The area of migration was annotated using GIMP (GNU Image Manipulation Program, Version 2.8) and determined using a selfprogrammed macro using Image J 1.46r (National Institutes of Health) software as published before ${ }^{19}$. The assay was performed in technical replicates $(n=8-11)$. A comparable experiment was conducted using $1.0 \times 10^{3}$ cells per spheroid $(n=10-12)$ showing analogous results.

\section{D-tumor spheroid-based invasion assay}

Upon spheroid formation after $72 \mathrm{~h}$, the invasive potential of tumor cells was analyzed by embedding the generated 3D-tumor spheroids in an artificial ECM. For this, compact spheroids were transferred into a 96-Well ULA round-bottom plate by pipetting $100 \mu \mathrm{L}$ (using a $1250 \mu \mathrm{L}$ pipette tip without filter on top of a $100 \mu \mathrm{L}$ pipette tip) of medium containing one single spheroid into each well. In this way, each well contained only one 
spheroid. When the spheroids were already generated in a 96-well ULA round-bottom plate it was sufficient to gently remove $100 \mu \mathrm{L}$ medium from each well. After thawing growth factor reduced Matrigel ${ }^{\circ}$ on ice, the 96well plate was placed on ice for $5 \mathrm{~min}$ to cool the wells. In this way an early polymerization of the Matrigel ${ }^{\circledR}$ was prevented. Then, $100 \mu \mathrm{L}$ Matrigel ${ }^{\oplus}$ were dispensed carefully into each well and mixed with the remaining medium by slowly pipetting up and down several times without introducing air bubbles into the mixture. To ensure that the spheroids were in a central position, the plates were centrifuged at $300 \mathrm{~g}$ for $3 \mathrm{~min}$ at $4^{\circ} \mathrm{C}$. The plates were then placed in an incubator at $37^{\circ} \mathrm{C}$ for $1 \mathrm{~h}$ until the Matrigel ${ }^{\oplus}$ had polymerized. Afterwards, $100 \mu \mathrm{L}$ of complete growth medium was added to each well. The plates were incubated at $37^{\circ} \mathrm{C}$ and $5 \% \mathrm{CO}_{2}$ for up to $96 \mathrm{~h}$. Tumor invasion was documented by taking pictures with an inverted light microscope in the brightfield channel at $\times 4$ and $\times 10$ magnification every $48 \mathrm{~h}$.

\section{NanoString sample preparation and nCounter assay}

Gene expression analysis was performed using the human nCounter PanCancer Progression Panel (NanoString Technologies, Hamburg, Germany). Total RNA was isolated from frozen cell pellets ( $48 \mathrm{~h}$ culture) by QIAzol-chloroform extraction followed by RNeasy Mini Kit (Qiagen, Hilden, Germany) preparation. Isolated RNA $(100 \mathrm{ng})$ was processed through the NanoString nCounter Prep Station. Briefly, the hybridization reaction $(3 \mu \mathrm{l}$ Reporter CodeSet, $5 \mu \mathrm{l}$ hybridization buffer, $2 \mu$ l Capture ProbeSet and $5 \mu$ l total RNA) containing $100 \mathrm{ng}$ RNA (HCT116: $n=3$; clone 7/6: $n=2$; clone 10/8: $n=3$; clone 21/9: $n=1$ ) was conducted for $16 \mathrm{~h}$ at $65^{\circ} \mathrm{C}$. Subsequently, samples were processed according to the manufacturer's instructions and signals of reporter probes were counted and tabulated using the nCounter Digital Analyzer (NanoString Technologies, Hamburg, Germany). Finally, housekeeping gene (geometric mean of 30 genes) normalization for quantitating gene expression levels, positive control normalization for background noise correction and data analysis was performed using the nSolver $^{\mathrm{TM}}$ Analysis Software 3.0 (NanoString Technologies, Hamburg, Germany) and standard settings. The foldchange of counts was determined by averaging results per DAPK1 ko cell line and comparing them to average counts of HCT116 cells. Only significant $(P<0.05)$ fold changes $>-1.5$ or $>+1.5$ fold were considered as differentially expressed in DAPK1 ko clones compared to the wildtype. Transcripts with a RNA count of $<5$ of all samples were excluded as they were considered as nonexpressed. For statistical analysis of fold changes, $p$ values of pairwise t-tests calculated by nSolver ${ }^{\mathrm{TM}}$ were consulted. Expression profiling data are available online (GEO accession number: GSE130488).

\section{Quantitative real-time PCR}

Total RNA from fresh-frozen cell line pellets (biological triplicates) and fresh xenograft tumors (3 tumors per cell line) was isolated using QIAzol and the RNAeasy Mini Kit (both from Qiagen, Hilden, Germany). For cDNA synthesis, $1 \mu \mathrm{g}$ RNA and the QuantiTect ${ }^{\circ}$ Reverse Transkription Kit (Qiagen, Hilden, Germany) were used. Quantitative real-time PCR analysis was performed using the QuantiTect SYBR Green RT PCR (Qiagen, Hilden, Germany) on the thermocycler CFX96 ${ }^{\mathrm{TM}}$ and the RealTime System $\mathrm{C} 1000^{\mathrm{TM}}$ (BioRad, Munich, Germany). Gene-specific primer pairs were: $B$-2-microglobuline (b2m)-sense: 5'-GAC TTG TCT TTC AGC AAG GA-3'; b2m-antisense: 5'-ACA AAG TCA CAT GGT TCA CA3'; icam1-sense: $5^{\prime}$-aag gtg acc gtg aat gtg ct-3'; icam1antisense: $5^{\prime}$-cgc tgg cgg tta tag agg ta-3'; tacstd2-sense: 5'-TCC CCT TTC GGT CCA ACA AC-3'; tacstd2antisense: 5'-AAA CGA TCC CGG GTT GTC AT-3'. For data analysis, raw counts were normalized to $\mathrm{b} 2 \mathrm{~m}$ gene $\left(\Delta \mathrm{Ct}=\mathrm{CT}_{\text {target }}-\mathrm{CT}_{\text {reference }}\right)$. Fold induction to HCT116 cell line was calculated using the $2^{-\Delta \Delta \mathrm{CT}}$ method with $\Delta \Delta \mathrm{Ct}=\Delta \mathrm{CT}_{\text {experimental }}-\Delta \mathrm{CT}_{\text {control. }}$ Each sample was analyzed in triplicates $(n=9)$.

\section{Gene list enrichment analysis}

Gene list enrichment analysis of significantly up- and down-regulated transcripts in DAPK1 ko clones vs. HCT116 wt were performed using the Enrichr tool ${ }^{21}$ and the integrated KEGG 2016 and REACTOME 2016 geneset databases. Lists of either significantly upregulated $(n=22)$ or down-regulated transcripts $(n=12)$ were used as input and only gene sets with an adjusted $P$ value $<0.05$ were considered significantly enriched.

\section{Calculation of the stromal scores for RNAs deregulated by DAPK1 ko in HCT116 cells}

A "stroma score" for RNAs with either "unaltered" (absolute fold change $<1.05$ ), "down-regulated" (fold change $<1.25$ ) or "upregulated" (fold change $>1.25$ ) expression between DAPK1 ko clones and HCT116 wildtype cells were calculated to illustrate that up and down-regulated RNAs in DAPK1 ko clones have more frequent stromal cell origin (in CRC tumors) as compared to unaltered transcripts, which are primarily of epithelial origin, i.e. deregulated transcripts are likely to affect tumor microenvironment ("cell extrinsic") rather than "cell intrinsic" processes. The "stroma scores" were calculated using a similar approach as in Bramsen et al. ${ }^{22}$ and is the fraction of mouse (i.e. stroma cell derived) vs. human (i.e. epithelial cancer cell derived) transcripts in human PDX tumors from mice evaluated by RNA sequencing by Isella et al. ${ }^{23}$ (used scores are listed in Supplementary Table 1). Hereby, a high and low stroma score indicate that transcripts are primarily of stroma cell 
(mouse) or cancer epithelial (human) origin in CRC tumors, respectively.

\section{Pre-ranked gene-set enrichment analysis (GSEA) of the DAPK1 ko expression signature}

Pre-ranked GSEA was performed using the GSEA V3.0 software ${ }^{24,25}$ using standard settings and all gene sets included in the Molecular Signatures Database v6.2. As input we used expression fold change values (DAPK1 ko clones vs. HCT116) for the 770 transcripts profiled by the nCounter PanCancer Progression Panel (NanoString Technologies Hamburg, Germany). Gene sets were manually categorized into functional gene-set groups representing biological properties relevant to this paper using the following keywords for each category: ECM matrix (keywords: matrisome, ECM, extracellular collagen); EMT/invasion (keywords: mesenchymal, invasive, epithelial cell migration); integrin pathways (keyword: integrin); cell-substrate adhesion (keywords: matrix adhesion, substrate adhesion, focal adhesion); and healing/ inflammation (keywords: wound, "inflam"); Cell-cell adhesion (keywords: cell cycle); chromosome organization (keywords: chromosome, chromatin). All gene sets containing the terms "positive regulation of", "Negative regulation of", "UP" and "DN" were omitted from the analysis.

\section{Nearest template prediction (NTP) classification of TCGA COREA samples and comparison to their CMS classification status}

CRC samples from the TCGA project was classified into three categories "DAPK1 ko up sign.", DAPK1 ko down-sign.", and DAPK1 ko unaltered sign." using the NTP module ${ }^{26}$ of GenePattern 2.027. As input we used RNA sequencing profiles for 434 COREAD samples acquired from the UCSC XENA Public Data Hubs (https://xena.ucsc.edu/public-hubs/) as $\log 2(\mathrm{FPKM}+$ 1) normalized RNA expression values for 20.530 genes. As classification templates we used the transcripts with either "unaltered" (absolute fold change < 1.05), "downregulated" (fold change $<-1.25$ ) or "upregulated" (fold change $>1.25$ ) expression between DAPK1 ko clones versus HCT116 wildtype cell line. NTP classifications were considered robust for samples with a BenjaminiHochberg $<0.05$. Consensus molecular subtype calls for the COREAD samples were acquired provided by the Colorectal Cancer Subtyping Consortium ${ }^{28}$ (CRCSC; "CMS final network plus RFclassifier in non-consensus samples". The analysis was restricted to CRC samples for which a CMS annotation was provided by the CRCSC.

\section{ECM cell adhesion assay}

The adhesion capacity of DAPK1 ko cells to different protein substrates was analyzed using the ECM Cell
Adhesion Array Kit, colorimetric (Merck, Millipore, Darmstadt, Germany) according to the manufacturer's recommendations. Briefly, after rehydration of the plate strips, $0.5 \times 10^{6}$ cells per 96 well were plated in triplicates per cell line and incubated for $1 \mathrm{~h}$ at $37^{\circ} \mathrm{C}$ and $5 \% \mathrm{CO}_{2}$. Thereafter, the supernatant was carefully removed, cells were washed with PBS and $100 \mu \mathrm{l}$ per well of Cell Stain Solution was added. After an incubation of $5 \mathrm{~min}$ at RT, cells were washed with $\mathrm{ddH}_{2} \mathrm{O}$ and were solubilized for 15 min in $100 \mu \mathrm{l}$ Extraction Buffer. The absorbance was then quantified at $570 \mathrm{~nm}$ in the multilabel reader VIC$\mathrm{TOR}^{\mathrm{TM}} \mathrm{X} 3$ (Perkin Elmer, Rodgau, Germany). BSAcoated wells served as negative control. The data were expressed as BSA-corrected absorbances as percent increase of HCT116 cells.

\section{Second-harmonic generation (SHG) microscopy}

We applied SHG microscopy in freshly harvested CAM tissues 3-5 days after tumor cell implantation using an upright multiphoton microscopy system (TriM-Scope II; LaVision BioTec GmbH, Bielefeld, Germany). A femtosecond Ti:sapphire laser was used for imaging at the excitation wavelength $800 \mathrm{~nm}$. The CAM was brought into focus using an HC Fluotar L25x/0.95 W Visir water immersion objective (Leica, Munich, Germany).

\section{STRING bioinformatic analysis}

For interpretation of the newly discovered DAPK1 ko gene expression pattern, protein-protein interactions and the interplay with the DAPK1-ERK2 axis were investigated using STRING interaction database (https://stringdb.org/).

\section{Immunofluorescence staining}

For immunofluorescence (IF) studies, $8.0 \times 10^{4}$ cells grown in $\mu$-slides 8 well (Ibidi, Martinsried, Germany) were fixed with $4 \%$ formaldehyde for $30 \mathrm{~min}$ at RT, permeabilized with IF buffer (PBS supplemented with $0.2 \%$ Triton X-100 and 0.05\% Tween20) and then blocked with $1 \%$ BSA in IF buffer for $30 \mathrm{~min}$ at RT. After washing the cells with PBS-glycine (100 mM glycine in PBS) they were incubated with primary antibodies anti-DAPK1 (1:80, BD Biosciences, Heidelberg, Germany) or anti-phosphoERK1/2 ${ }^{\text {Thr202/Tyr204 }}$ (1:80, Cell Signaling, Frankfurt am Main, Germany) diluted in IF buffer at $4{ }^{\circ} \mathrm{C}$ overnight. Cells were washed with PBS and incubated with appropriate fluorochrome-conjugated secondary antibodies AlexaFluor 488 (1:500, A-11029) or AlexaFluor 555 (1:500, A-21428) (both Thermo Fisher Scientific, Waltham, MA, USA) at RT for $2 \mathrm{~h}$. Following another washing step, cells were counterstained with Hoechst 33342 (5 $\mu \mathrm{g} /$ $\mathrm{ml}$, Sigma-Aldrich, Taufkirchen, Germany) for DNA staining and optionally with Alexa Fluor 568 Phalloidin (1:40 of $\times 40$ stock, A12379; Thermo Fisher Scientific, 
Waltham, MA, USA) to visualize cytoskeletal F-actin. Representative images were captured using a fluorescence microscope Nikon Eclipse Ti using 100x oil immersion objective lens (both from Nikon, Düsseldorf, Germany). Confocal images for phospho-ERK1/2 ${ }^{\text {Thr202/Tyr204 }}$ were obtained using a confocal laser scanning microscopy system (LSMT-PMT Observer ZI, LSM 710, Carl Zeiss Inc, Oberkochen, Germany) with a $\times 63$ oil objective. Images were background corrected using Image analysis software.

\section{Transient siERK2 transfection experiment}

To obtain an ERK2 knockdown, HCT116 and DAPK1 ko clone $7 / 6$ and $21 / 9$ cells were grown to $70 \%$ confluence in a 6 well culture plate and transfected with DharmaFECT reagent and $100 \mathrm{nM}$ of siRNA (SMARTpool: ONTARGETplus Human MAPK3 (ERK2) siRNA (both from Dharmacon, Lafayette CO, USA) according to the manufacturer's instructions and incubated for 48 and $72 \mathrm{~h}$. Transfection with non-targeting SMARTpool siRNA served as negative control. The knockdown efficiency was determined by Western Blotting. ERK2 knockdown experiments were repeated in two independent experiments and representative Western blots are shown.

\section{Statistical analysis}

All statistical tests (using two-tailed Mann-Whitney test, one-way ANOVA multiple comparison, unpaired $t$ test, multiple t-test and Pearson's correlation) were performed using Prism 7 (San Diego, California, USA). Differences were considered statistically significant according to values of two-tailed ${ }^{*} P<0.05,{ }^{* *} P<0.01,{ }^{* * *} P<0.001$. Types of the tests are indicated in the figure legends.

\section{Results \\ Evaluation of HCT116-derived CRISPR/Cas9-mediated DAPK1 ko clones}

Basal level of DAPK1 protein in the HCT116 cell line was determined using Western blot (Fig. 1a). HCT116 express low to moderate DAPK1 compared to DLD1 and HT29, which express high levels of DAPK1, and in comparison to SW480, SW620 and SW837, where DAPK1 levels were not detected (Fig. 1a). To mirrorimage the loss of DAPK1 protein observed at tumor invasion front of colorectal tumors, we first established CRISPR/Cas9-driven DAPK1 ko HCT116 cell lines using two different single guide RNAs (sgRNA1 and 2) (Supplementary Fig. 1a, b). The presence of Cas9 protein, which serves as evidence for an active CRISPR/ Cas9 system was shown in the lysate of HCT116 cells $24 \mathrm{~h}$ after transient transfection with sgRNA1 or sgRNA2 vectors by Western Blot whereas it was no longer detectable in the established monoclonal ko cell clones (Supplementary Fig. 1c). Sanger sequencing analysis revealed homozygeous and sgRNA-specific insertion mutations resulting in a reading frame shift (Supplementary Fig. 1d). Three monoclonal DAPK1 ko cell lines named clone $7 / 6,10 / 8$ and 21/9 were randomly chosen from a panel of several successfully generated subclones and the lack of endogenous DAPK1 protein was verified by immunofluorescence staining (Fig. 1b; Supplementary Fig. 2a) and Western Blotting (Fig. 1c). To exclude CRISPR/Cas9-caused off-target effects on other highly related DAPK family members, we determined the protein expression of DAPK2 (DRP-1), DAPK3 (ZIPK), DRAK1 and DRAK2 in Western Blotting (Fig. 1c). Steady state protein levels in HCT116 cells and in 10/8 and 21/9 revealed no ko effects on these molecules. The protein levels of pMLC, a well known DAPK1 target, were decreased in the DAPK1 ko clones (Fig. 1c). Regarding "stemness", we found differences between the single clones with clone 7/6 had the highest expression of CD133 and CD44 markers and clone 21/9 did not express CD44 at all (Fig. 1d). No epithelial-to-mesenchymal transition (EMT) was observed in DAPK1 clones visualized by steady E-cadherin protein levels and lack of vimentin expression (Fig. 1d).

In confocal immunofluorescence images we showed that pERK1/2 in HCT116 cells is predominantly localized in the cytoplasm (Fig. 1e), whereas in the three DAPK1 ko clones pERK $1 / 2$ expression was remarkably increased in the nuclei of tumor cells in vitro (Fig. 1e). These findings were confirmed analyzing nuclear and cytoplasmatic protein fractions in Western Blot (Fig. 1f) indicating that the loss of DAPK1 is associated with nuclear pERK1/ 2 shuttling under endogeneous conditions.

The functional DAPK1 ko was verified in a 3D migration spheroid system. We evaluated the whole areas since the border of the cores were not well defined. At day 4 we confirmed an anti-migratory role of DAPK1 in all three clones (Supplementary Fig. 3a, b). Moreover, in agreement with our previous findings ${ }^{29}$ with DAPK1 as a proapoptotic player, the TNF- $\alpha$ induced phosphorylation of Cofilin was remarkably diminished in DAPK1 ko clones (Supplementary Fig. 3c). In summary, these findings strongly support the functional ko in our newly generated DAPK1 ko cell lines.

\section{In vivo growth pattern of DAPK1 ko clones}

Next, we used the CAM assay to examine the role of DAPK1 loss for tumor growth. DAPK1 ko clones and parental HCT116 cells were transplanted onto the chicken CAM and were cultured in ovo for 5 days as schematically illustrated in Fig. 2a. Examples of CAM xenografts are shown in Fig. 2b. Conventional HE staining of HCT116-derived CAM tumors reflected the typical growth pattern of a microsatellite instable colorectal carcinoma with well-differentiated epithelial-like 


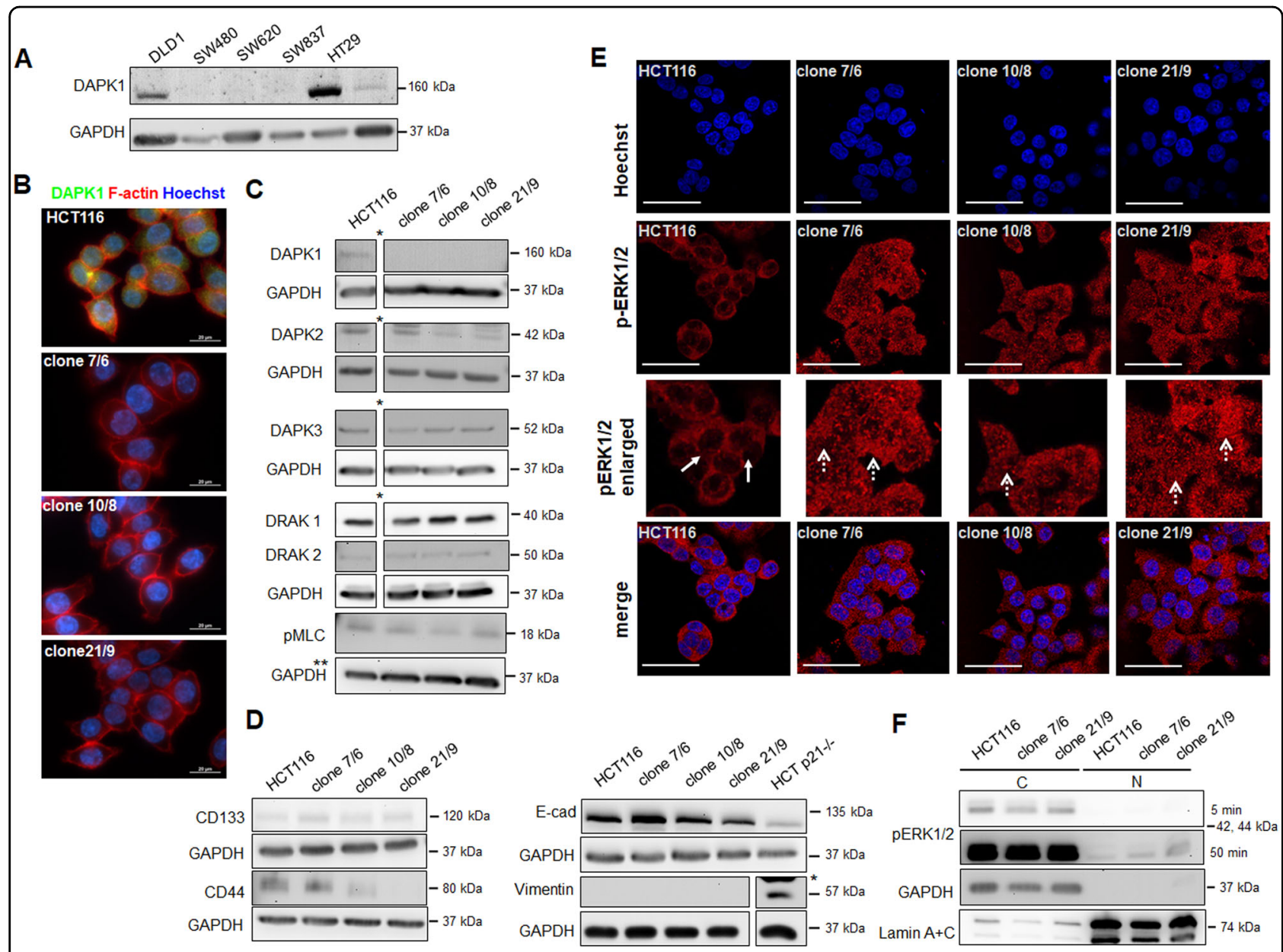

Fig. 1 Validation of CRISPR/Cas9-mediated DAPK1 knockout in HCT116 colorectal cancer cells. a Western Blot of DAPK1 expression status in various colorectal cancer cell lines. Representative images of two independent experiments are shown. GAPDH served as loading control. b Immunofluorescence staining of DAPK1 (green) in parental HCT116 cells and DAPK1 ko clones. Cells were counterstained with phalloidin for F-actin (red) and nuclear Hoechst (blue). Fluorescence microscopy was performed using a x 100 oil immersion objective. Representative images of two independent experiments are shown. Scale bar $=20 \mu \mathrm{m}$. c Protein expression of DAPK1 family members DAPK1, DAPK2, DAPK3, DRAK1, DRAK2 and DAPK1 phosphorylation target pMLC in HCT116 wildtype cells and DAPK1 ko clones were detected by Western Blotting using specific primary antibodies. Representative images of two independent experiments are shown. * images were cropped here; all samples were analyzed on the same SDS-PAGE gel; ** GAPDH blot has been used twice see Fig. 5 b, proteins have been loaded on the same membrane. $\mathbf{d}$ Western Blot analysis of stem cell markers (CD133, CD44) and EMT markers (epithelial marker: E-cad = E-cadherin, mesenchymal marker: Vimentin). Representative images of two independent experiments are shown.*images were cropped here; all samples were analyzed on the same SDS-PAGE gel. e Representative images of endogenous phospho-ERK1/2 (red) levels of immunostained HCT116 cells and DAPK1 ko clones examined by confocal immunofluorescence microscopy (63x; enlarged: cropped and zoomed in). Cells were nuclear counterstained with Hoechst (blue). Immunofluorescence was repeated in two independent experiments and representative images are shown. White arrows: empty nucleus; dashed arrow: nuclear expression of pERK1/2. Scale bar $=50 \mu \mathrm{m}$. $\mathbf{f}$ pERK1/2 expression analyzed by Western Blot in cytoplasmic (C) and nuclear (N) protein fractions. Representative images of two independent experiments are shown. GAPDH served as loading control in total and cytoplasmatic protein fractions. Lamin A/C was used for nuclear loading control.

glandular structures and clear margins pushing back the chicken connective tissue (Fig. 2c). In contrast, DAPK1deficient tumors showed a shift to loosely packed tumor masses and a highly infiltrative growth pattern intruding the CAM (Fig. 2c).

Pan-CK staining of CAM xenografts was used to identify human CRC cells in the chicken mesodermal layer and to highlight tumor budding (Fig. 2d). The avian epithelial monolayers were also Pan-CK positive. Quantification of peritumoral budding, single cells or small clusters of up to four cells ahead of the invasive front ${ }^{10}$, revealed a trend of an increase in budding in DAPK1 ko tumors at day 5 (not significant, Fig. 2e). When evaluating a budding score with low-grade budding defined as an average of $\leq 1$ and highgrade budding as $>1$ buds per 10 high-power-fields (HPFs; $\times 40$ ), the DAPK1-dependent difference was remarkable. 


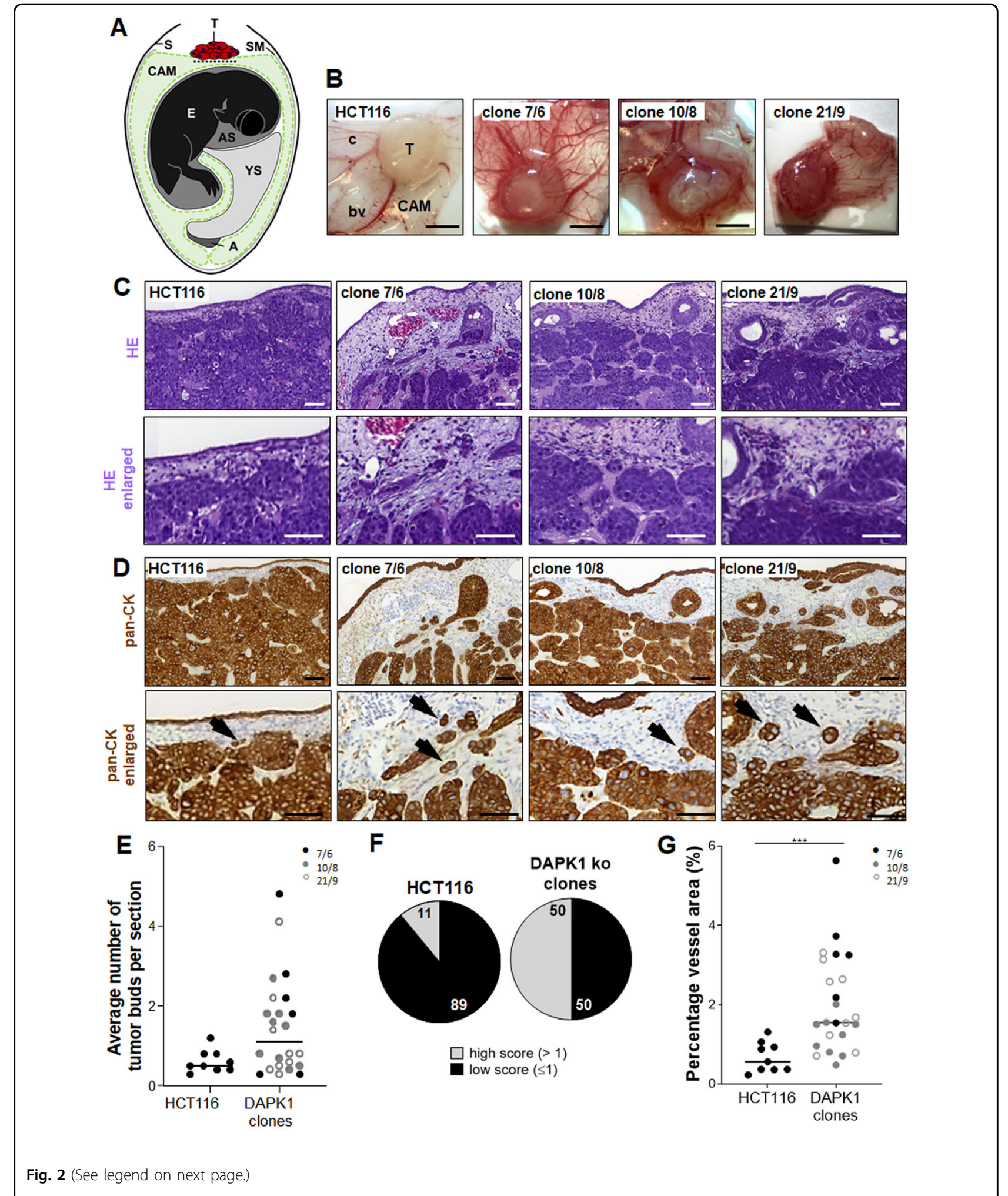

DAPK1 loss was associated with high-grade tumor budding (50\% of evaluated tumors) demonstrating that every second HPF of DAPK1 negative tumors was given a high- grade score whereas $89 \%$ of all HCT116 wildtype tumors were low-grade budders (Fig. 2f). Since CAM assay is a classical in vivo model for angiogenesis, we analyzed 


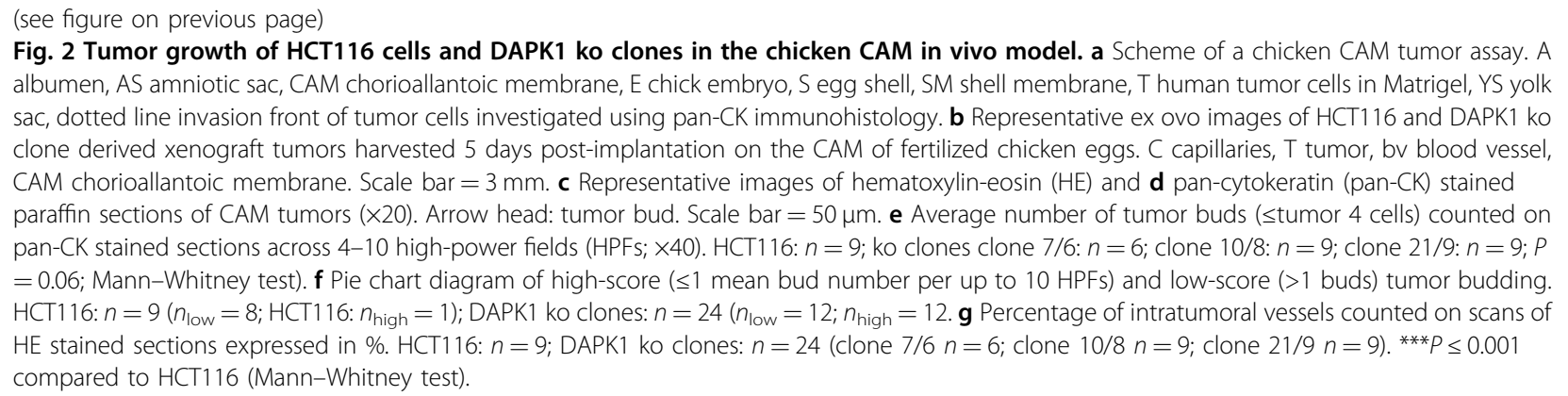

neovascularization in HCT116 and DAPK1 ko tumors. DAPK1 ko tumors showed higher vessel area compared to HCT116 (Fig. 2g; Supplementary Fig. 4) indicating DAPK1 being an anti-angiogenic factor in CRC. Proliferation did not differ in a DAPK1-dependent manner in vitro (Fig. 3a) and in vivo when evaluating the $\mathrm{pHH} 3$ positive tumor cell population (Fig. 3b, c) in the CAM model which is in close agreement with the rather similar tumor volumes of CAM xenografts (Fig. 3d).

For in vitro analysis of invasive potential of HCT116 and DAPK1 ko clones 7/6, 10/8 and 21/9 tumor cells, 3Dtumor spheroids were embedded in matrigel and cell penetration from the spheroid core into the environment was monitored over time (Fig. 4a). Even though none of the cell lines developed any invadopodia-like spikes, we observed outgrowth of compact protrusions in HCT116, clone $10 / 8$ and $21 / 9$ after $48 \mathrm{~h}$ and even more pronounced bulges after $96 \mathrm{~h}$. Clone 7/6, however, failed to develop protrusions. Applying semi-automated quantification of the invasion area, we investigated that DAPK1 ko clones $10 / 8$ and $21 / 9$ exhibited significantly enhanced $(* * * * P<$ 0.001 ) invasive capacity compared to HCT 116 after $96 \mathrm{~h}$, while clone $7 / 6$ revealed significantly smaller invasive areas after $48 \mathrm{~h}\left({ }^{*} P<0.05\right)$ and $96 \mathrm{~h}\left({ }^{* *} P<0.01\right.$; Fig. $\left.4 \mathrm{~b}\right)$.

Next we confirmed the highly invasive behavior of DAPK1 ko tumor cells in another 3D ex vivo model, where tumor cells were applied and cultured on precision-cut tissue slices of rat brain (Fig. 4c). These brain slices at least partly reflect the complexity of an organotypic environment. Tracking green fluorochromelabeled tumor cells, we found larger areas of invasion for DAPK1 ko clones one day after transplantation reflecting their increased invasive capability in a physiological 3D matrix in contrast to HCT116 wildtype cells $\left({ }^{*} P<0.05\right.$; ${ }^{* *} P<0.01$; Fig. 4c, d). Although a high variability, significance levels were reached for all three ko clones at day one. However, at day three after transplantation, tumor areas reached nearly equal fluorescence intensities (Fig. 4e) suggesting that DAPK1 ko cells have a better early adaptation capability to the 3D environment. Notably, just like the CAM tumors (Fig. 2c), HCT116 cells generated dense tumor masses while DAPK1 ko clones formed more loosely packed and more spacious tumors as reflected by a more diffuse green fluorescence (Fig. 4c). PI staining of empty brain slices and slices with transplanted HCT116 and DAPK1 ko cells for quality control showed equal mean intensities of PI signal at day 1 and day 3 of the experiment (Supplementary Fig. 5a). The slight decrease in tumor area at day 3 might be caused by a loss of fluorescent dye due to proliferation of cells.

Furthermore, we examined if the increase in tumor budding was associated with an enhanced potential to form metastases in the chicken embryonic organs. Since DAPK1 loss is associated with anoikis resistance ${ }^{30}$, we hypothesized that DAPK1 ko tumor cells might have a survival benefit in the vascular system reflected by a higher number of disseminating tumor cells in the embryonic chicken organs. To test this hypothesis, prelabeled HCT116 and DAPK1 ko clone 21/9 tumor cells were transplanted onto the CAM and whole bodies of freshly sacrificed chicken embryos were screened for optical signals using an intravital imaging system (IVIS Spectrum, Perkin Elmer; Fig. 4f, Supplementary Fig. 5b). Clone $21 / 9$ seems to be representative since it showed more migration, more invasion and an elevated number of budding. Results exposed significantly higher average radiant efficiency $(* * P<0.01)$ within animals loaded with clone 21/9-derived DAPK1 ko tumors (Fig. 4f) suggesting that DAPK1 ko cells showed more disseminating tumor cells, preferentially accumulating in the liver, heart, and brain of the chicken embryo.

\section{Identification of DAPK1 ko-related gene expression signature}

For determination of the DAPK1-dependent gene expression signature, we performed a NanoString-based gene expression analysis. Our panel covered genes which are involved in ECM remodeling, epithelial-mesenchymal transition (EMT), metastasis, and angiogenesis, some major hallmarks crucial for tumor progression. Parental HCT116 cells and each of the three DAPK1 ko clones revealed high similarity in their overall gene expression 

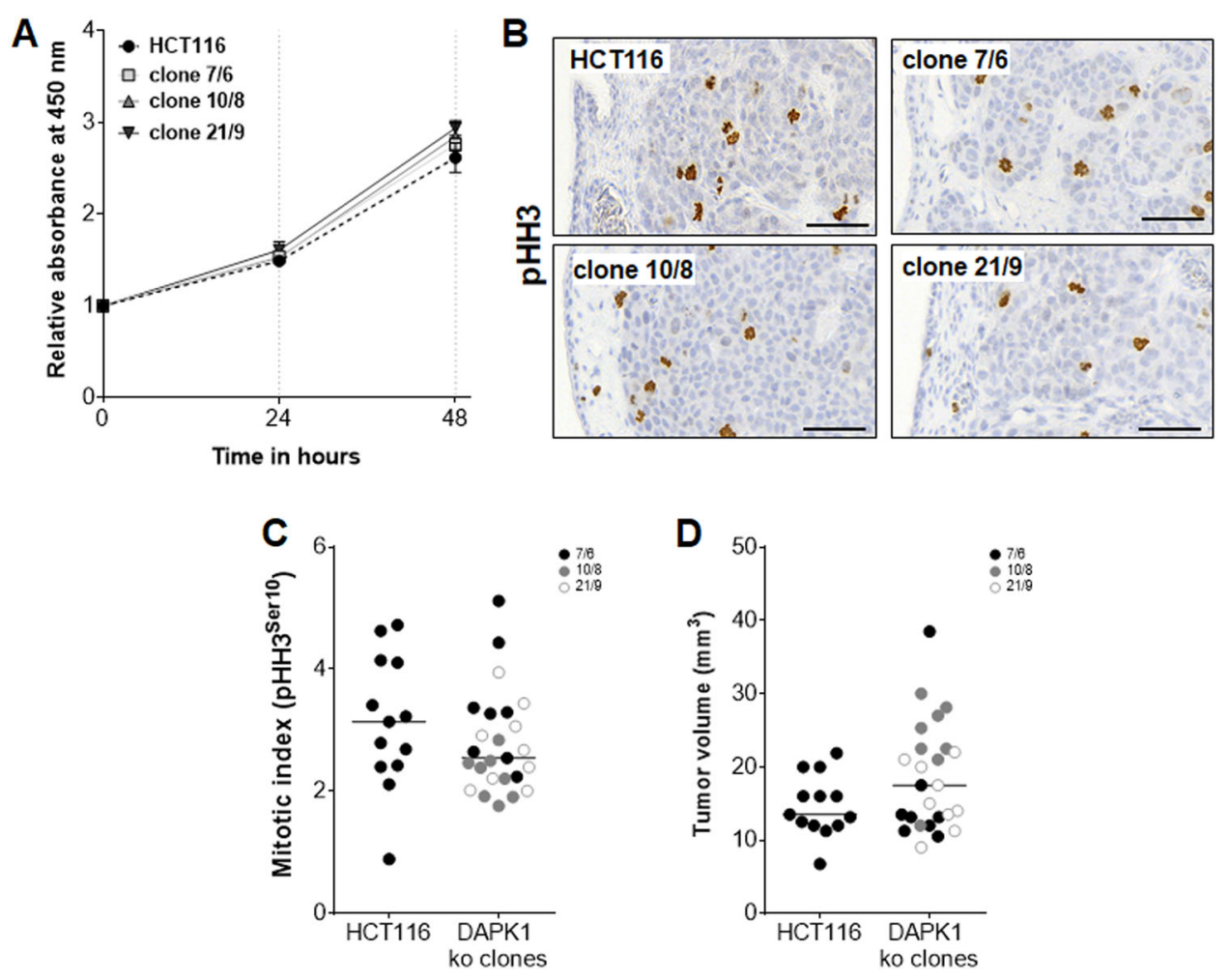

Fig. 3 The proliferative capacity of HCT116 cells and DAPK1 ko clones in vitro and in vivo and CAM tumor volumes. a WST-8 assay of HCT116 cell and DAPK1 ko clones 7/6, 10/8 and 21/9 was conducted for quantification of the metabolic activity after pre-incubation of 0, 24 and $48 \mathrm{~h}$ and $2 \mathrm{~h}$ of WST-8 treatment. Absorbance was measured at $450 \mathrm{~nm}$ and presented as a relative growth curve with respect to time-point $=0 \mathrm{~h}$. The mean of two independent experiments ( $n=4$ replicates per experiment) is shown, ( $P>0.5$; two-way ANOVA) $\mathbf{b}$ Immunohistochemical staining of mitoses in CAM tumor sections derived from HCT116 cells and DAPK1 ko clones using $\mathrm{pHH} 3^{\text {Ser10 }}$ specific primary antibody. Representative images are shown. Scale bar $=50 \mu \mathrm{m}$. c The mitotic rate of cells in CAM tumors was determined using pHH3 immunohistochemistry. Quantification of mitoses per $10 \mathrm{HPFs}$ per section ( $\times 20)$ was performed with QuPath software (https://qupath.github.io/). The average number of mitoses per section is presented as dot. Medians of the data are presented as lines in the scatter plots. (HCT116: $n=13$; clone 7/6: $n=8$; clone 10/8: $n=8$; clone 21/9: $n=9$ $(P=0.1884$; Mann-Whitney test). $\mathbf{d}$ Volumes of 5-day-old CAM xenograft tumors were measured and calculated as follows assuming an ellipsoid shape: $V_{\text {Tumor }}=$ length $\times$ width $\times$ height $\times \pi / 6$ and presented as dots. Medians of the data are presented as lines in the scatter plots. HCT116: $n=13 ;$ clone 7/6: $n=8$; clone 10/8: $n=8$; clone 21/9: $n=9$ ( $P=0.1652$; Mann-Whitney test).

signature (Pearson's $r=0.99, \quad * * * P<0.0001$; data not shown). When screening for genes similarly altered in all three DAPK1 ko cell lines 7/6, 10/8 and 21/9, a significant increase (>1.5 fold) in IL11, TACSTD2, CLEC2B, P3H2, LAMA4, CCL5, SPDEF, CHRDL1, SERPINE1, COL5A1, HEG1, AGRN, TGFB2, COL7A1, GALNT7, PLAUR, CGN, ITGA1, NOTCH1, TBXA2R, LAMA5 and TNS1 and decrease (>1.5 fold) in JAG1, CALCRL, GPR124, SCG2, INHBE, CADM1, ACVRL1, COL6A3, FGF9, AGT, ANGPT2 and ICAM1 were found (Table 1). Exemplarily, we selected TACSTD2 and ICAM1 as interesting candidates upon DAPK1 loss since they are majorly involved in ECM interaction and metastasis. We confirmed the endogenous TACSTD2 up- and ICAM1 downregulation on gene expression and protein levels using qPCR analysis (Fig. 5a) and Western Blotting (Fig. 5b) in all DAPK1 ko cell clones. The same effects on TACSTD2 and ICAM1 were confirmed in snap-frozen DAPK1 ko CAM tumors when analyzing mRNA expression $\left({ }^{*} P<0.05\right.$; ${ }^{* *} P<0.01$; Fig. 5c) and protein levels using Western Blot (Fig. 5d). ICAM1 loss and TACSTD2 gain were also validated in vivo in DAPK1 ko CAM tumors by immunohistochemistry $\left({ }^{* *} P<0.01\right.$; Fig. 5 e). Finally, we could show that TACSTD2 expression was dramatically enhanced in all DAPK1 ko clones 7/6, 10/8 and 21/9 $\left(^{* * * * *} \mathrm{P}<0.0001\right)$ compared to HCT116 in CAM xenografts (Fig. 5e).

We inspected deregulated genes to identify biological processes possibly affected by DAPK1 loss in the ko clones. Foremost, gene list enrichment analysis of the 34 significantly deregulated transcripts suggested that they were involved in regulating tumor-stroma interactions, in particular altered ECM organization and matrix adhesion was characteristic for upregulated transcripts (Fig. 6a). In agreement with a role in tumor-stroma 


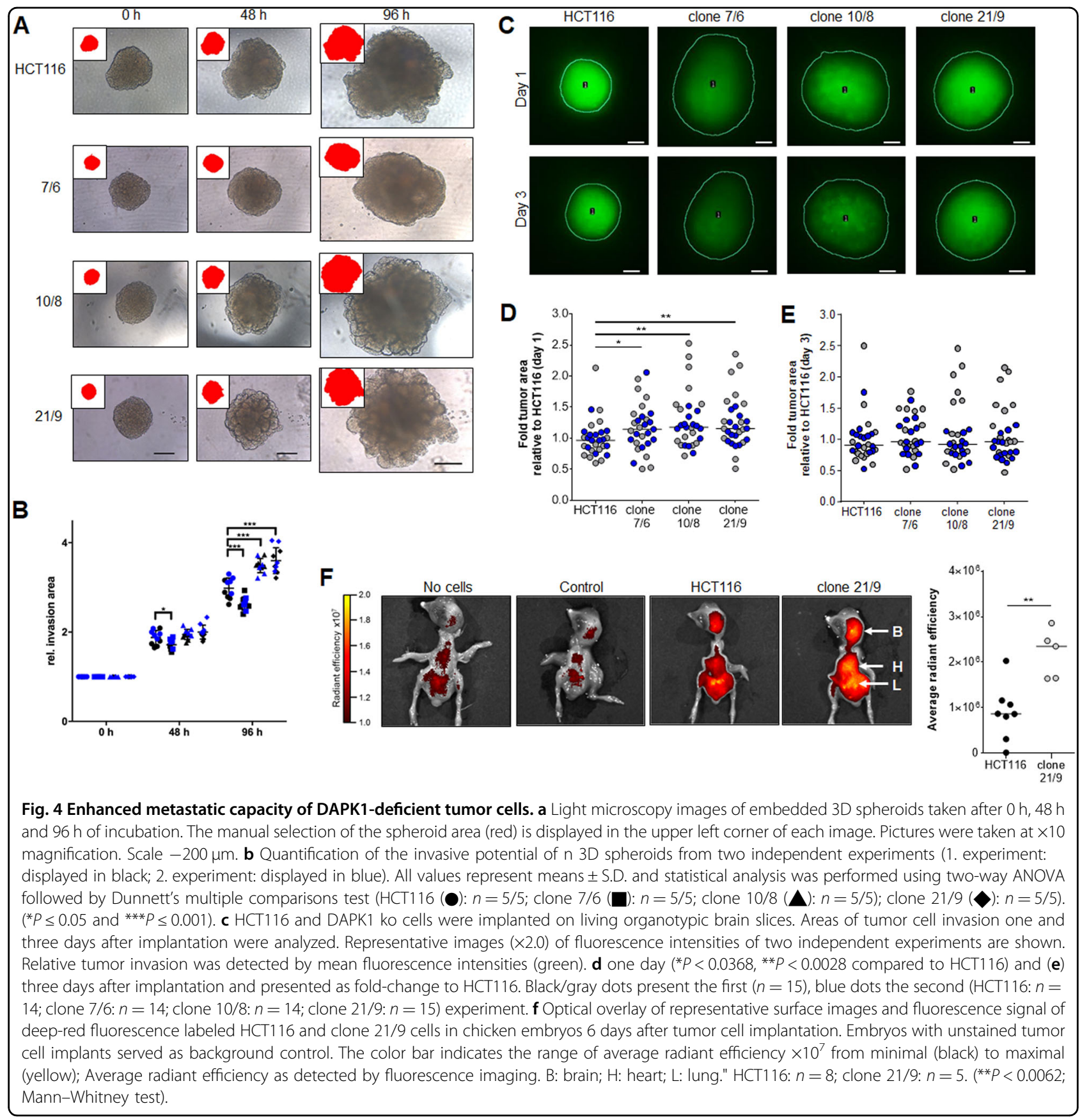

modulation, we found that both sets of up- and downregulated transcripts had a significantly higher "stroma score" (i.e. transcripts primarily expressed by nonepithelial cells within the tumor stroma) than unaffected genes when analyzing all transcripts (Fig. 6b). To closer investigate which tumor-stroma processes were up- and down-regulated upon DAPK1 ko we performed preranked gene-set enrichment analysis (pre-ranked GSEA) using all gene sets in the BROAD Database (MsigDB) gene-set collection v6.2. Handpicked gene sets representing the biological properties that correlate with the DAPK1 ko phenotype in Fig. 6c. We found that gene sets for organization and binding to ECM and mesenchymal transition had higher Normalized Enrichment Scores (NESs) in DAPK1 ko clones, whereas gene sets for immune pathways, cell cycle and chromosome/chromatin organization had negative NESs (Fig. 6c). Collectively, these analyses suggested that loss of DAPK1 in HCT116 cells affected transcripts involved in enhancing tumor-stroma binding and mesenchymal transition. 
Table 1 Fold change difference of mRNA expression of DAPK1 ko clones versus HCT116.

\begin{tabular}{|c|c|c|c|c|}
\hline Reference sequence & Gene ID & Gene name & $\begin{array}{l}\text { Average fold change } \\
\text { (log2) } \pm \text { SEM }\end{array}$ & $\begin{array}{l}P \text {-value } \\
(t \text {-test) }\end{array}$ \\
\hline \multicolumn{5}{|l|}{ A: upregulated genes } \\
\hline NM_000641.2 & IL11 & Interleukin-11 & $5.91 \pm 0.76$ & $<0.001$ \\
\hline NM_002353.2 & TACSTD2 & Tumor-associated calcium signal transducer 2 & $5.13 \pm 3.12$ & 0.004 \\
\hline NM_005127.2 & $C L E C 2 B$ & C-type lectin domain family 2 member B & $4.71 \pm 7.14$ & 0.016 \\
\hline NM_018192.2 & P3H2 & Prolyl 3-hydroxylase 2 & $2.78 \pm 0.64$ & 0.007 \\
\hline NM_001105209.1 & LAMA4 & Laminin, alpha 4 & $2.31 \pm 0.47$ & 0.031 \\
\hline NM_002985.2 & CCL5 & Chemokine ( $C-C$ motif) ligand 5 & $2.25 \pm 0.33$ & 0.05 \\
\hline NM_012391.1 & SPDEF & SAM pointed domain containing ets transcription factor & $2.22 \pm 0.43$ & 0.031 \\
\hline NM_001143981.1 & CHRDL1 & Chordin-like 1 & $2.22 \pm 0.36$ & 0.011 \\
\hline NM_001165413.1 & SERPINE1 & $\begin{array}{l}\text { Serpin peptidase inhibitor, clade E (nexin, plasminogen activator inhibitor } \\
\text { type 1), member } 1\end{array}$ & $2.07 \pm 0.34$ & 0.001 \\
\hline NM_000093.3 & COL5A1 & Collagen, type V, alpha 1 & $1.95 \pm 0.96$ & 0.024 \\
\hline NM_020733.1 & HEG1 & Protein HEG homolog 1 & $1.78 \pm 0.28$ & 0.003 \\
\hline NM_198576.2 & AGRN & Agrin & $1.75 \pm 0.16$ & $<0.001$ \\
\hline NM_003238.2 & TGFB2 & Transforming growth factor, beta 2 & $1.73 \pm 0.36$ & 0.012 \\
\hline NM_000094.2 & COL7A1 & Collagen, type VII, alpha 1 & $1.63 \pm 0.85$ & 0.037 \\
\hline NM_017423.2 & GALNT7 & $\begin{array}{l}\text { UDP-N-acetyl-alpha-D-galactosamine:polypeptide N- } \\
\text { acetylgalactosaminyltransferase } 7 \text { (GalNAC-T7) }\end{array}$ & $1.57 \pm 0.03$ & $<0.001$ \\
\hline NM_001005376.1 & PLAUR & Plasminogen activator, urokinase receptor & $1.57 \pm 0.10$ & $<0.001$ \\
\hline NM_020770.2 & CGN & Cingulin & $1.57 \pm 0.32$ & 0.034 \\
\hline NM_181501.1 & $|T G A|$ & Integrin, alpha 1 & $1.56 \pm 0.32$ & 0.049 \\
\hline NM_017617.3 & NOTCH1 & Neurogenic locus notch homolog protein 1 & $1.55 \pm 0.17$ & 0.021 \\
\hline NM_001060.3 & TBXA2R & Thromboxane A2 receptor & $1.52 \pm 0.21$ & 0.038 \\
\hline NM_005560.3 & LAMA5 & Laminin subunit alpha-5 & $1.5 \pm 0.17$ & 0.006 \\
\hline NM_022648.4 & TNS1 & Tensin 1 & $1.5 \pm 0.19$ & 0.011 \\
\hline \multicolumn{5}{|l|}{ B: down-regulated genes } \\
\hline NM_000214.2 & $J A G 1$ & Jagged 1 & $-1.58 \pm 0.34$ & 0.035 \\
\hline NM_005795.3 & CALCRL & Calcitonin receptor-like & $-1.62 \pm 0.04$ & 0.006 \\
\hline NM_032777.9 & GPR124 & G protein-coupled receptor 124 & $-1.73 \pm 0.13$ & 0.023 \\
\hline NM_003469.3 & SCG2 & Secretogranin ॥ & $-2.2 \pm 1.13$ & 0.043 \\
\hline NM_031479.3 & INHBE & Inhibin, beta $\mathrm{E}$ & $-2.28 \pm 0.62$ & 0.016 \\
\hline NM_014333.3 & CADM1 & Cell adhesion molecule 1 & $-2.42 \pm 0.57$ & 0.049 \\
\hline NM_000020.1 & ACVRL1 & Activin A receptor type II-like 1 & $-2.53 \pm 0.40$ & 0.009 \\
\hline NM_004369.3 & COL6A3 & Collagen, type Vl, alpha 3 & $-2.62 \pm 0.57$ & 0.004 \\
\hline NM_002010.2 & FGF9 & Fibroblast growth factor 9 & $-2.88 \pm 1.78$ & 0.042 \\
\hline NM_000029.3 & $A G T$ & Angiotensinogen (serpin peptidase inhibitor, clade A, member 8) & $-2.88 \pm 0.50$ & 0.035 \\
\hline NM_001147.2 & ANGPT2 & Angiopoietin 2 & $-8.02 \pm 1.44$ & 0.002 \\
\hline NM_000201.2 & ICAMI & Intercellular adhesion molecule 1 & $-9.94 \pm 1.88$ & $<0.001$ \\
\hline
\end{tabular}

Gene expression analysis was performed using the human $n$ Counter ${ }^{\oplus}$ PanCancer Progression Panel (NanoString Technologies, Hamburg, Germany). Only significant $(P<0.05)$ fold changes $>-1.5$ or $>+1.5$ fold were considered as differentially expressed in DAPK1 ko clones compared to the wildtype. Transcripts with an RNA count of $<5$ of all samples were excluded as they were considered as non-expressed. The average of clones (clone 7/6: $n=2$; clone 10/8: $n=3 ;$ clone 21/9: $n=1$ ) vs. HCT116 $(n=3) \pm$ is presented. $P$ value was calculated by $n$ Solver software (Nanostring Technologies) using $t$-test. Expression profiling data are available online (GEO accession number: GSE130488)

Notably, classification of TCGA CRC samples (COREAD) using NTP suggested that the aggressive CMS4 subtype tumors were indeed associated with the gene signature upregulated in the DAPK1 ko clones as compared to down- or unaffected gene signatures (Fig. 6d).

To support the suggestion that ECM interaction was altered in DAPK1 ko clones we performed an ECM cell adhesion array, which revealed that DAPK1 ko cells significantly benefit from loss of DAPK1 regarding binding to collagen I, II and IV ("$P<0.05,{ }^{* *} P<0.01$; ${ }^{* *} P<0.01$; Fig. 6e). Indeed, SHG images of the surrounding CAM showed differences in collagen alignment for HCT116 and DAPK1 ko tumors. Whereas there were areas of very similar collagen alignment as found in the control CAM without xenografts (Fig. 6f-1 versus 2,6), DAPK1 ko engrafted CAMs showed a higher variation in the extend and arrangement of collagen fibers. DAPK1 ko was majorly associated with a more regular and polarized collagen fiber structure (Fig. 6f-7,8), abundant thicker bundles (Fig. $6 \mathrm{f}-$ $5,7,8$ ) with network building (Fig. 6f-5,7,8). In CAMs 


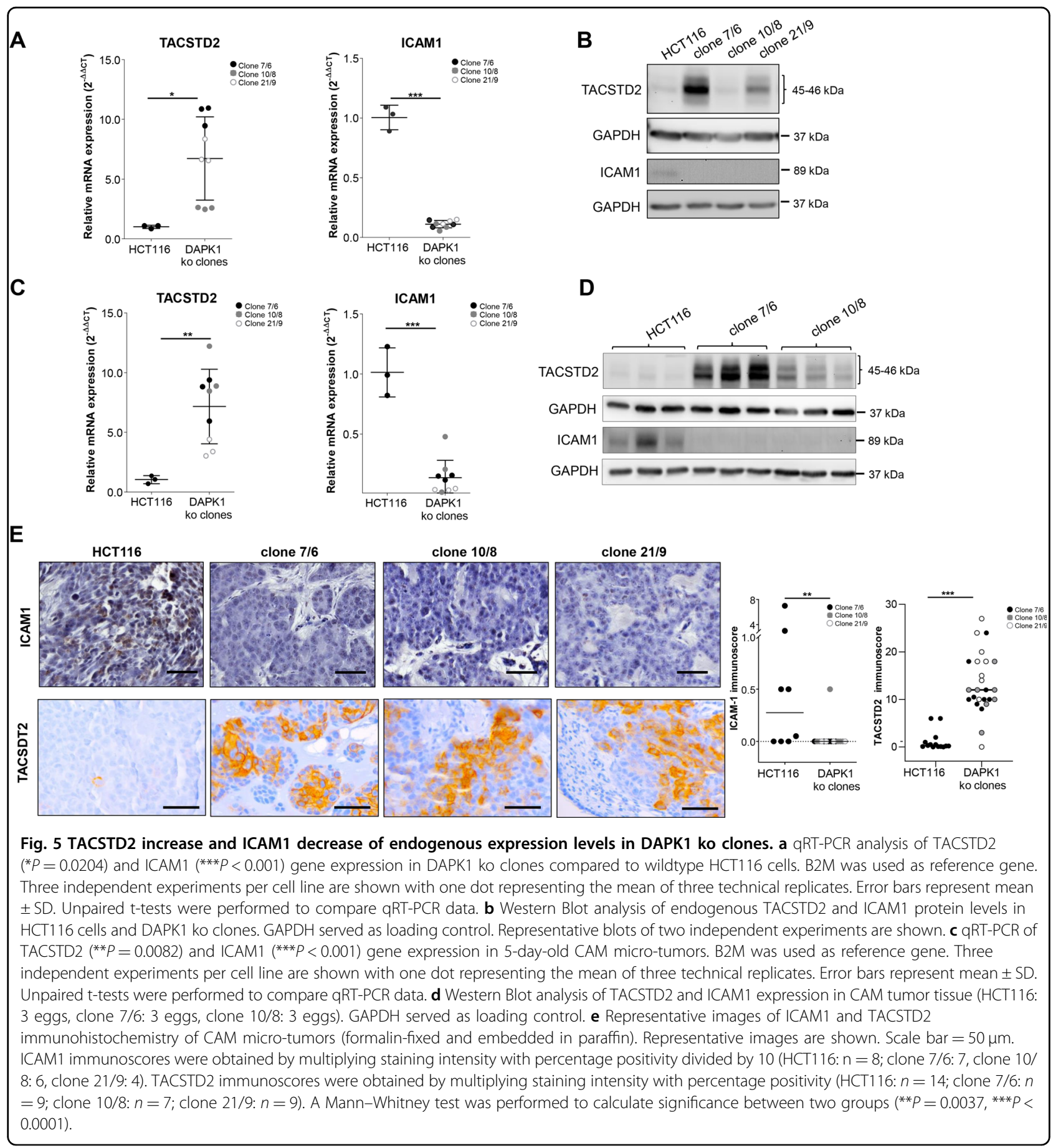

engrafted with HCT tumors we could hardly detect any aligned fibers in the matrix and the fibers were oriented in a multitude of directions (Fig. $6 f-2-4$ ).

\section{DAPK1/ERK2 signaling axis targets TACSTD2 expression}

It has been reported that ERK when phosphorylating DAPK1 at Ser ${ }^{735}$ induces a negative feedback loop since DAPK1's death domain is then binding to ERK, blocking its nuclear translocation and finally triggering apoptosis induction ${ }^{31}$. To analyze oncogenic players of the DAPK1-ERK2 mediated regulation axis, we performed a STRING analysis including the 34 deregulated DAPK1-dependent genes (Table 1) and added DAPK1 and ERK2 (MAPK3) into this network (Fig. 7). Indeed, ERK2 was situated in close neighborhood of DAPK1 (Fig. 7). The network itself consisted of two major 


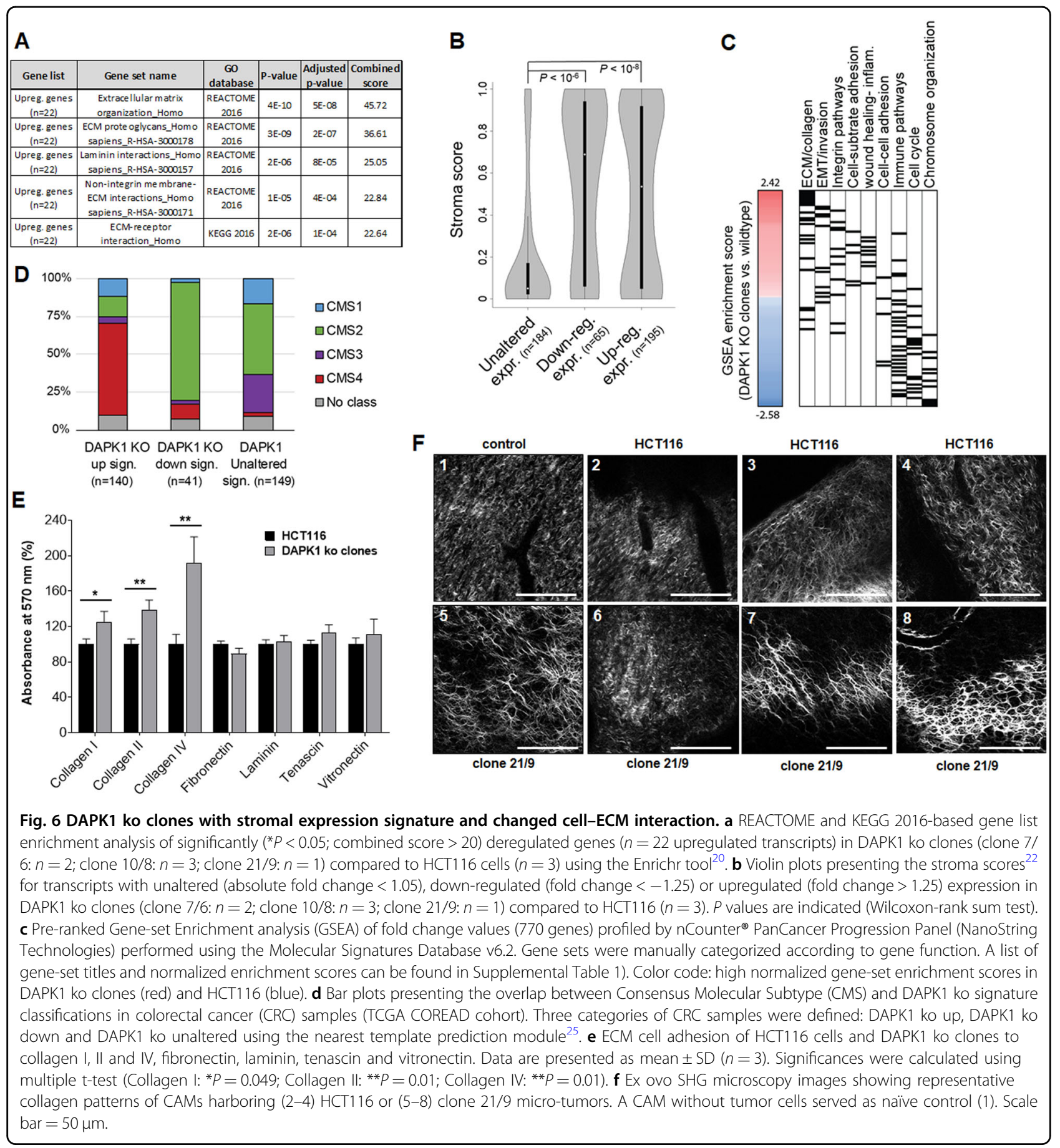

nodules: NOTCH1 and ECM modulators. Interestingly, DAPK1 seems to regulate the network from outside linking the two clusters. Notably, so far, CLEC2B, Heg1, GALNT7, CALCRL, GPR124, CGN, and TACSTD2 have not been studied for their association with the DAPK1 network. The DAPK dependent gene signature involved six different dysregulated ECM components (Fig. 7).
As expected, pERK $1 / 2$ protein was mainly localized in the nuclei when evaluating CAM xenografts of DAPK1 ko clones (Fig. 8a-c). Inhibition of ERK1/2 kinase activity using 10 and $20 \mu \mathrm{M}$ of ERK1/2 inhibitor (FR180204) in HCT116 slightly increased cleaved PARP and ICAM1 levels and decreased TACSTD2 levels (Fig. 8d). Next, we inhibited ERK2 signaling by siERK2 transfection of HCT116, clone 21/9 and 7/6 cells (Fig. 8e). An ERK2 


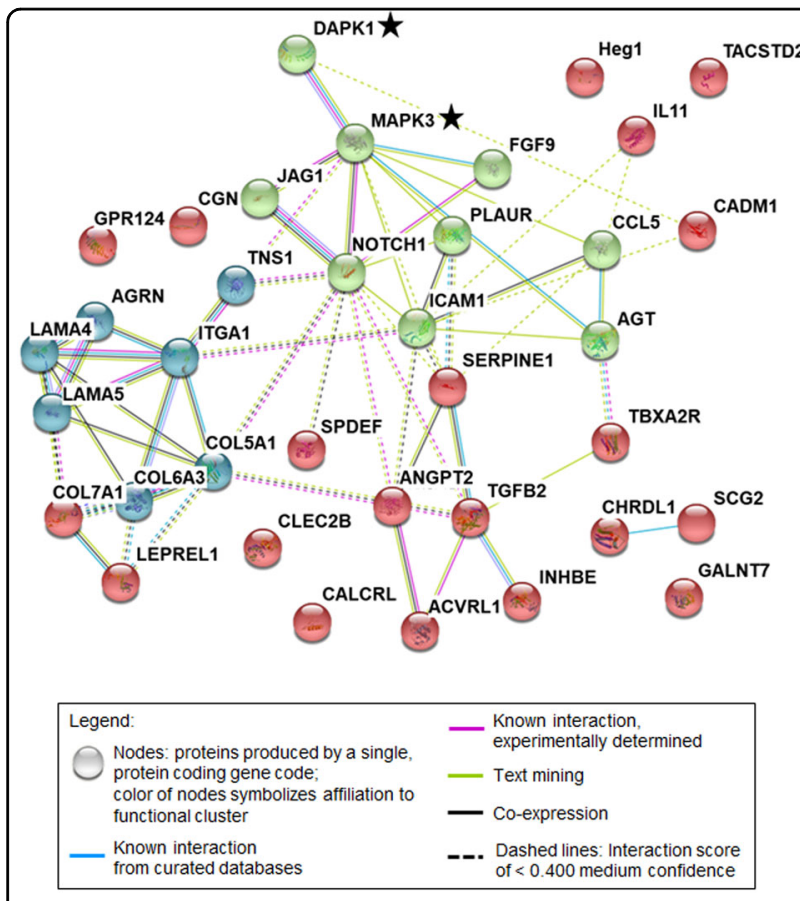

Fig. 7 Visualization of suggested DAPK1/MAPK3(ERK2)/ICAM1/ TACSTD2 signaling found via STRING database search of top 34 dysregulated genes of DAPK1 ko panel. Black star: additional input to STRING analysis. Color scheme: green: Notch1 cluster, blue: cluster of ECM modulators, red: cluster of more distant interactions. For full gene names and gene reference please refer to Table 1.

inhibition Western Blot analysis showed decreased TACSTD2 levels in HCT116 cells and in 21/9 cells whereas ICAM1 remained undetectable (Fig. 8e) suggesting that DAPK1-ERK2 axis might be partly involved in regulation of TACSTD2, but not in regulation of ICAM1.

\section{Discussion}

In this study, we firstly report a novel DAPK1-mediated network associated with metastatic potential of colorectal tumors. We show that under DAPK1 loss, colon tumor cells gain ability to modulate the ECM consequently increasing their metastatic potential in vitro and in vivo. We hypothesize that this hallmark of cancer could be partly driven by the DAPK1-ERK axis.

So far, there have been only a few hints obtained from CRC patient data suggesting that DAPK1 functionally acts as a metastasis suppressor in the colonic epithelium. It has been shown in two independent studies that DAPK1 expression was decreased in tumors that had already metastasized at time of diagnosis ${ }^{3,4}$. In papillary thyroid cancer, pituitary tumors, hepatocellular, and esophageal carcinoma DAPK1 promoter hypermethylation was correlated with advanced tumor stages and worse prognosis $^{32-35}$. The molecular basis of these observations has only been little understood.
Here, we give novel experimental evidence for DAPK1's role in migration, invasion, and ECM modulation and antimetastatic potential in CRC cells. DAPK1 seems to exert sensor functions under conditions where an interaction with ECM and basement membrane components is possible. Our study required a long-term and stable gene knockout which was implemented by the CRISPR/Cas9 technology. Slight differences in molecular and functional readouts among the randomly picked DAPK1 ko clones are caused by the monoclonal strategy which was applied to the heterogeneous HCT116 parental cell line. Thus, as a result of mixed or inefficient gene editing (a per-cell rate of $30-60 \%$ CRISPR ko is reported), an efficient conversion to an uniform cell population which was required for proper gene ko assessment, can be reached only by singlecell cloning ${ }^{36}$. Although a complete loss of DAPK1 expression was verified, we showed that the genetic background of single clones was varying especially regarding stem cell marker expression. Such genetic background pattern might modify the DAPK1 signature, however, can be mostly neglected in our approach since we only focused on genes that were commonly deregulated in all three DAPK1 clones.

We identified a DAPK1-driven network of 22 genes upregulated under loss of DAPK1 in HCT116 cells. Literature research (Pubmed, September 2019) revealed that 73\% (IL11, TACSTD2, CLEC2B, LAMA4, CCL5, SERPINE1, TGFB2, COL7A1, GALNT7, PLAUR, CGN, ITGA1, NOTCH1, TBXA2R, LAMA5, TNS1) of upregulated genes found in our study have already been described in previous studies in CRC. In particular, IL11 promotes growth of neoplastic epithelium ${ }^{37}$, and is associated with poor differentiation, a large tumor size, lymph node metastasis and overall low survival of CRC patients $^{38}$. CLEC2B belongs to C-type lectins which facilitate the tumor metastasis in many cancers ${ }^{39}$. Interestingly, two laminins LAMA4 and LAMA5 were upregulated in DAPK1 ko signature ${ }^{40,41}$. LAMA5 is required for growth of hepatic metastases where it promotes branching angiogenesis and regulates Notch signaling ${ }^{42}$. In accordance with the observed increased collagen binding in vitro and in vivo in DAPK1 ko clones, ITGA1, which encodes the alpha 1 subunit of integrin receptors (collagen surface receptor), has been found to be upregulated in the DAPK1 ko clones. Moreover, the negative regulator of the MMP-associated proteolytic network, the serine protease inhibitor SERPINE1 (plasminogen activator inhibitor-1) seemed to be associated with aggressive tumor behavior in $\mathrm{CRC}^{43,44}$. It was reported to maintain an angiogenic "scaffold" and stabilizes nascent capillary structure which is in agreement with the observed proangiogenic phenotype under DAPK1 loss.

For 23\% (P3H2, CHRDL1, COL5A1, HEG1, AGRN) DAPK1 dysregulated genes their role in CRC is 


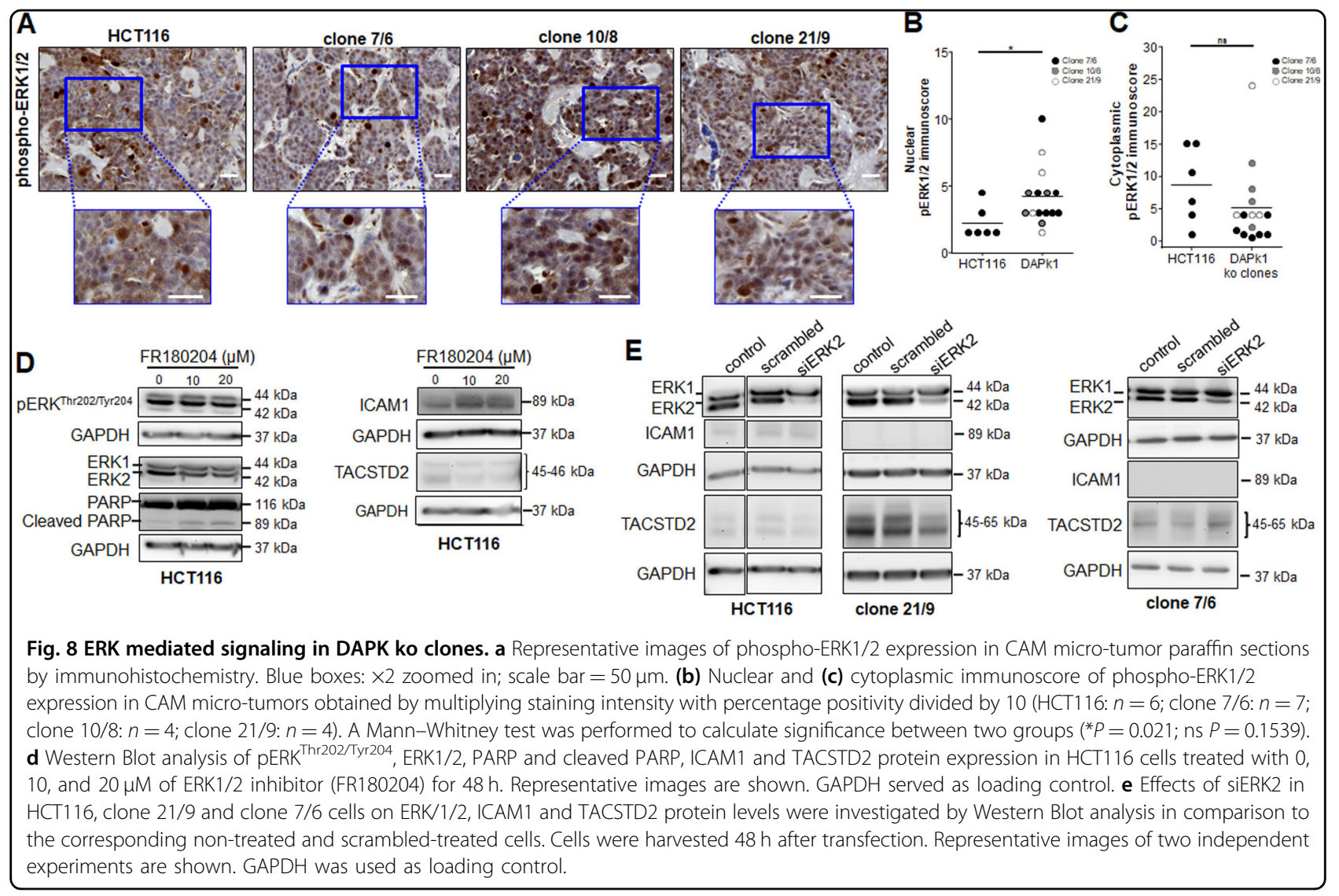

completely unknown. Taken together, the identified overall gene expression signature in the DAPK1 ko cell lines confirms our hypothesis, that DAPK1 might inhibit CRC progression and metastasis.

Then we focused further on TACSTD2 (TROP2), a cell surface receptor that transduces $\mathrm{Ca}^{2+}$ signals. Although TACSTD2 was found to be highly expressed in many cancer types, only a few very recent reports are available about its functional role in cancer ${ }^{45}$. TACSTD2 has been described as an oncogene promoting cell proliferation, epithelial-to-mesenchymal transition, and metastasis in bladder and colon cancer ${ }^{46,47}$. EpCAM positive circulating breast cancer cells were enriched for TACSTD2 expression linking it to a higher metastatic capability of tumor cells ${ }^{48}$. In our study, we showed in vitro and in vivo that DAPK1 loss led to higher TACSTD2 expression in an ERKdependent manner. Thus, one of the tumor suppressor functions of DAPK1 might be partly mediated by suppressing the metastasis associated TACSDT2. Loss of DAPK1 in tumor buds at tumor invasion front of CRC as we previously described ${ }^{4}$ would consequently add more aggressive properties to disseminating tumor cells. This hypothesis is supported by findings that TACSTD2 promotes cell motility in prostate cancer cells by modulating the $ß 1$ integrin signaling and increases wound healing by promoting stem cell survival ${ }^{49,50}$. DAPK1 is known to inactivate integrin $\beta 1$ dependent matrix survival signals and TACSTD2 would potentiate this signaling, in a DAPK1 loss situation also further reducing the integrin-dependent ICAM1 signaling. Indeed we found an up-regulation of integrin $\beta 1$ expression (1.22-fold, $P=0.07$ ) when DAPK1 is lost. During metastatic cascade, tumor cells interact not only with several immune cells from the tumor environment, but also with fibronectin, laminin, and type I collagen of the basement membrane. In our study, we showed for the first time that the loss of DAPK1 was associated with a higher collagen binding. The reorganized collagen fiber meshwork of the CAM around DAPK1 ko xenografts seems to trigger cell invasion and distant metastasis formation. This might also explain the better and faster adaptation of DAPK1 loss tumor cells in the physiological environment of PCTS experiment. DAPK1 loss might promote dissemination seen as increase in number of tumor buds and remodeling of the CAM collagen matrix triggering the colonization of tumor cells at a secondary site since we found significantly more metastasis in chicken embryonic organs from DAPK1 ko xenografts.

Tumor progression with metastasis is associated with severe alterations in cell-cell and cell-matrix interactions. One of the genes remarkably deregulated under DAPK 
loss was found to be the cell surface protein ICAM1. Here we describe a role for DAPK in regulating ICAM1 and subsequently in interaction with ECM components of the tumor microenvironment. Indeed, we showed that the loss of DAPK1 and ICAM1 was associated with an upregulation of integrins that are specifically interacting with collagens. Since under DAPK1 loss both ICAM1 transcripts and ICAM1 protein are completely lost we believe in a transcriptional regulation mechanism. ICAM1 has been shown mainly to act in different cell types as a metastasis suppressor ${ }^{51-53}$. A lower number of ICAM-1 positive cells has been observed in metastasizing $\mathrm{CRC}^{54}$. Under conditions where ICAM1 is lost, the tumor microenvironment is remarkably rebuilt and an increased M2 polarization of macrophages has been observed ${ }^{55}$ leading to maintenance of acute inflammation and an attenuated tissue repair ${ }^{56}$. There is still controversy about the role of ICAM1 in tumor metastasis since high ICAM1 expression has been described in advanced melanoma ${ }^{57}$. So ICAM1 might fulfill different functional properties dependent on the cellular subtype and the specific pathologic situation. DAPK1 has been shown to be inactivated by Netrin- $1^{58}$ to potentiate tumor-associated vessel formation in metastatic lung cancer ${ }^{59}$. Through modulating the tumor-associated vasculature DAPK might also be involved in shaping the tumor microenvironment.

We have identified a connection between DAPK1, ICAM1 and MAP kinase ERK1/2 in STRING analysis. DAPK1 is a phosphorylation substrate of ERK and vice versa. When DAPK1 is phosphorylated by ERK at Ser735 (interacting via its death domain) it holds ERK in the cytoplasm $^{31}$, thus preventing its nuclear translocation. This reciprocal regulation leads to a positive feedback loop that promotes apoptosis. Thus, we speculate that endogenous DAPK1 loss could lead to an increase in nuclear shuttling of active pERK1/2. Indeed we observed more pERK $1 / 2$ protein in the nucleus in $2 \mathrm{D}$ immunofluorescence, in cell fractionation, and in vivo in CAM xenografts of DAPK1 ko clones. Since ERK has to translocate into the nucleus to regulate gene transcription, cell proliferation and differentiation ${ }^{60}$, we suggest that DAPK1 loss at the invasion front of CRC should have to do at least in part with an ERK1/2 triggered signaling cascade. Since inhibition of ERK signaling did not affect ICAM1 expression level we suggest that ERK signaling is not majorly involved in DAPK1-mediated ICAM1 expression.

In summary, we have successfully modeled the in vivo situation that DAPK1 is mostly lost at the tumor invasion front of CRC. We suggest a novel and even more comprehensive picture of DAPK1's antimetastatic functions by giving the first time experimental data that it diminishes an effective tumor cell-ECM interaction. DAPK1 exerts its tumor suppressor function at least partly via suppressing the metastasis associated TACSDT2. The potential of the
DAPK1 loss gene signature for metastasis prediction and therapy resistance should be further investigated.

\section{Acknowledgements}

The present work was performed in partial fulfillment of the requirements for obtaining the degree Dr. rer. nat. for SS at the Friedrich-Alexander University Erlangen-Nuremberg. The authors of the manuscript would like to thank Adrian Koch (AK) and Ingrid Mons (IM) for contributing experimental data, Christa Winkelmann (CW) and Rudolf Jung (RJ) for providing help in

immunohistochemistry and paraffin sectioning, Nicole Fuhrich and Thao Van for technical support in preparing histological scans and Prof. Jeffrey Parvin (OSU, Columbus/OH, USA) for providing his expertise in CRISPR/Cas9 approach. This article is partly based upon work from COST Action CA17118, which was supported by COST (European Cooperation in Science and Technology, www. cost.eu). The study was partly supported by a grant of the Interdisciplinary Center of Clinical Research of the University Hospital of the FAU Erlangen-Nürnberg (IZKF project D21). SS received an IZKF traveling grant in 2015. TB was partly supported by the German Research Foundation (DFG, INST 410/77-1, 410/93-1 FUGG).

\section{Author details}

${ }^{1}$ Experimental Tumor Pathology, University Hospital Erlangen, FriedrichAlexander University Erlangen-Nuremberg, Universitaetsstrasse 22, 91054 Erlangen, Germany. ${ }^{2}$ Institute of Pathology, University Hospital Erlangen, Friedrich-Alexander University Erlangen-Nuremberg, Krankenhausstr. 8-10, 91054 Erlangen, Germany. ${ }^{3}$ Experimental Trauma Surgery, Department of Trauma Surgery, University Regensburg Medical Center, Regensburg, Germany. ${ }^{4}$ Department of Molecular Medicine, Aarhus University Hospital, Palle JuulJensens Boulevard 99, 8200 Aarhus N, Denmark. Institute of Biotechnology, Friedrich-Alexander University Erlangen-Nuremberg, Paul-Gordan-Str. 3, 91052 Erlangen, Germany. ${ }^{6}$ Department of Cancer Biology and Genetics, College of Medicine, The Ohio State University Comprehensive Cancer Center, Columbus, $\mathrm{OH}$, USA. ${ }^{7}$ Department of Neurosurgery, University Hospital ErlangenNuremberg, Schwabachanlage 6, 91054 Erlangen, Germany. ${ }^{8}$ Department of Radiology, University Hospital Erlangen-Nuremberg, Maximiliansplatz 1, 91054 Erlangen, Germany

Conflict of interest

The authors declare that they have no conflict of interest.

\section{Publisher's note}

Springer Nature remains neutral with regard to jurisdictional claims in published maps and institutional affiliations.

Supplementary Information accompanies this paper at (https://doi.org/ 10.1038/s41419-019-2122-z).

Received: 17 May 2019 Revised: 3 October 2019 Accepted: 6 November 2019

Published online: 26 November 2019

\section{References}

1. Remmele, W. \& Stegner, H. E. [Recommendation for uniform definition of an immunoreactive score (IRS) for immunohistochemical estrogen receptor detection (ER-ICA) in breast cancer tissue. Pathologe 8, 138-140 (1987).

2. Gozuacik, D. et al. DAP-kinase is a mediator of endoplasmic reticulum stressinduced caspase activation and autophagic cell death. Cell Death Differ. 15, 1875-1886 (2008).

3. Chen, H. Y., Lee, Y. R. \& Chen, R. H. The functions and regulations of DAPK in cancer metastasis. Apoptosis 19, 364-370 (2014).

4. Ivanovska, J. et al. DAPK loss in colon cancer tumor buds: implications for migration capacity of disseminating tumor cells. Oncotarget 6, 36774-36788 (2015).

5. Schneider-Stock, R. Death-associated kinase (DAPK): a cancer "gene chameleon". Apoptosis 19, 285 (2014).

6. Cohen, O. et al. DAP-kinase participates in TNF-alpha- and Fas-induced apoptosis and its function requires the death domain. J. Cell Biol. 146, 141-148 (1999). 
7. Deiss, L. P., Feinstein, E., Berissi, H., Cohen, O. \& Kimchi, A. Identification of a novel serine/threonine kinase and a novel $15-\mathrm{kD}$ protein as potential mediators of the gamma interferon-induced cell death. Genes Dev. 9 15-30 (1995)

8. Inbal, B. et al. DAP kinase links the control of apoptosis to metastasis. Nature 390, 180-184 (1997)

9. Raveh, T., Droguett, G., Horwitz, M. S., DePinho, R. A. \& Kimchi, A. DAP kinase activates a p19ARF/p53-mediated apoptotic checkpoint to suppress oncogenic transformation. Nat. Cell Biol. 3, 1-7 (2001).

10. Lugli, A. et al. Recommendations for reporting tumor budding in colorectal cancer based on the International Tumor Budding Consensus Conference (ITBCC) 2016. Mod. Pathol. 30, 1299-1311 (2017).

11. Schneider-Stock, R. et al. Close localization of DAP-kinase positive tumourassociated macrophages and apoptotic colorectal cancer cells. J. Pathol. 209, 95-105 (2006).

12. Bialik, S. \& Kimchi, A. The death-associated protein kinases: structure, function, and beyond. Annu. Rev. Biochem 75, 189-210 (2006).

13. Mittag, F. et al. DAPK promotor methylation is an early event in colorectal carcinogenesis. Cancer Lett. 240, 69-75 (2006).

14. Li, H., Ray, G., Yoo, B. H., Erdogan, M. \& Rosen, K. V. Down-regulation of deathassociated protein kinase-2 is required for beta-catenin-induced anoikis resistance of malignant epithelial cells. J. Biol. Chem. 284, 2012-2022 (2009).

15. Castro, F. et al. High-throughput SNP-based authentication of human cell lines. Int J. Cancer 132, 308-314 (2013).

16. Cong, $L$. et al. Multiplex genome engineering using CRISPR/Cas systems. Science 339, 819-823 (2013).

17. Morgenstern, J. P. \& Land, H. Advanced mammalian gene transfer: high titre retroviral vectors with multiple drug selection markers and a complementary helper-free packaging cell line. Nucleic Acids Res. 18, 3587-3596 (1990).

18. Suzuki, K., Bose, P., Leong-Quong, R. Y., Fujita, D. J. \& Riabowol, K. REAP: a two minute cell fractionation method. BMC Res. Notes 3, 294 (2010).

19. Muenzner, J. K. et al. Generation and characterization of hepatocellular carcinoma cell lines with enhanced cancer stem cell potential. J. Cell Mol. Med 22, 6238-6248 (2018).

20. Karamitopoulou, E. et al. Proposal for a 10-high-power-fields scoring method for the assessment of tumor budding in colorectal cancer. Mod. Pathol. 26, 295-301 (2013).

21. Kuleshov, M. V. et al. Enrichr: a comprehensive gene set enrichment analysis web server 2016 update. Nucleic Acids Res. 44, W90-W97 (2016).

22. Bramsen, J. B. et al. Molecular-subtype-specific biomarkers improve prediction of prognosis in colorectal cancer. Cell Rep. 19, 1268-1280 (2017).

23. Isella, C. et al. Stromal contribution to the colorectal cancer transcriptome. Nat. Genet. 47, 312-319 (2015).

24. Subramanian, A. et al. Gene set enrichment analysis: a knowledge-based approach for interpreting genome-wide expression profiles. Proc. Natl Acad. Sci. USA 102, 15545-15550 (2005).

25. Barbie, D. A. et al. Systematic RNA interference reveals that oncogenic KRASdriven cancers require TBK1. Nature 462, 108-112 (2009).

26. Hoshida, Y. Nearest template prediction: a single-sample-based flexible class prediction with confidence assessment. PLOS ONE 5, e15543 (2010).

27. Reich, M. et al. GenePattern 2.0. Nat. Genet. 38, 500-501 (2006).

28. Guinney, J. et al. The consensus molecular subtypes of colorectal cancer. Nat. Med. 21, 1350-1356 (2015)

29. Ivanovska, J. et al. Identification of DAPK as a scaffold protein for the LIMK cofilin complex in TNF-induced apoptosis. Int. J. Biochem. Cell Biol. 45, 1720-1729 (2013).

30. Chang, C. C. et al. CCN2 inhibits lung cancer metastasis through promoting DAPK-dependent anoikis and inducing EGFR degradation. Cell Death Differ. 20, 443-455 (2013).

31. Chen, C. H. et al. Bidirectional signals transduced by DAPK-ERK interaction promote the apoptotic effect of DAPK. EMBO J. 24, 294-304 (2005).

32. $\mathrm{Hu}, \mathrm{S}$. et al. Association of aberrant methylation of tumor suppressor genes with tumor aggressiveness and BRAF mutation in papillary thyroid cancer. Int J. Cancer 119, 2322-2329 (2006).

33. Simpson, D. J., Clayton, R. N. \& Farrell, W. E. Preferential loss of death associated protein kinase expression in invasive pituitary tumours is associated with either CpG island methylation or homozygous deletion. Oncogene 21, 1217-1224 (2002)

34. Matsumoto, $\mathrm{H}$. et al. Prognostic significance of death-associated protein-kinase expression in hepatocellular carcinomas. Anticancer Res. 23, 1333-1341 (2003).
35. Bai, J. et al. Silencing DNA methyltransferase 1 (DNMT1) inhibits proliferation, metastasis and invasion in ESCC by suppressing methylation of RASSF1A and DAPK. Oncotarget 7, 44129-44141 (2016).

36. Ran, F. A. et al. Genome engineering using the CRISPR-Cas9 system. Nat Protoc. 8, 2281-2308 (2013).

37. Wu, J. et al. Transcriptional activation of FN1 and IL11 by HMGA2 promotes the malignant behavior of colorectal cancer. Carcinogenesis 37, 511-521 (2016).

38. Yoshizaki, A. et al. Expression of interleukin (IL)-11 and IL-11 receptor in human colorectal adenocarcinoma: $\mid \mathrm{L}-11$ up-regulation of the invasive and proliferative activity of human colorectal carcinoma cells. Int. J. Oncol. 29, 869-876 (2006).

39. Ding, D., Yao, Y., Zhang, S., Su, C. \& Zhang, Y. C-type lectins facilitate tumor metastasis. Oncol. Lett. 13, 13-21 (2017).

40. Chen, $M$. et al. Identification of a novel biomarker-CCL5 using antibody microarray for colorectal cancer. Pathol. Res. Pr. 215, 1033-1037 (2019).

41. Suenaga, M. et al. Serum VEGF-A and CCL5 levels as candidate biomarkers for efficacy and toxicity of regorafenib in patients with metastatic colorectal cancer. Oncotarget 7, 34811-34823 (2016).

42. Gordon-Weeks, A. et al. Tumour-derived laminin alpha5 (LAMA5) promotes colorectal liver metastasis growth, branching angiogenesis and notch pathway inhibition. Cancers (Basel) 11, https://doi.org/10.3390/cancers11050630 (2019).

43. Mazzoccoli, G. et al. ARNTL2 and SERPINE1: potential biomarkers for tumor aggressiveness in colorectal cancer. J. Cancer Res. Clin. Oncol. 138, 501-511 (2012).

44. Zeng, C. \& Chen, Y. HTR1D, TIMP1, SERPINE1, MMP3 and CNR2 affect the survival of patients with colon adenocarcinoma. Oncol. Lett. 18, 2448-2454 (2019).

45. Goldenberg, D. M., Stein, R. \& Sharkey, R. M. The emergence of trophoblast cell-surface antigen 2 (TROP-2) as a novel cancer target. Oncotarget $\mathbf{9}$, 28989-29006 (2018)

46. Li, X. et al. TROP2 promotes proliferation, migration and metastasis of gallbladder cancer cells by regulating PI3KVAKT pathway and inducing EMT. Oncotarget 8, 47052-47063 (2017).

47. Zhao, P. \& Zhang, Z. TNF-alpha promotes colon cancer cell migration and invasion by upregulating TROP-2. Oncol. Lett. 15, 3820-3827 (2018).

48. Schneck, H. et al. EpCAM-independent enrichment of circulating tumor cells in metastatic breast cancer. PLOS ONE 10, e0144535 (2015).

49. Trerotola, M. et al. Trop-2 promotes prostate cancer metastasis by modulating beta(1) integrin functions. Cancer Res. 73, 3155-3167 (2013).

50. Yang, J. et al. Trop2 regulates the proliferation and differentiation of murine compact-bone derived MSCs. Int J. Oncol. 43, 859-867 (2013).

51. Maeda, K. et al. Expression of intercellular adhesion molecule-1 and prognosis in colorectal cancer. Oncol. Rep. 9, 511-514 (2002).

52. Ogawa, Y. et al. Expression of intercellular adhesion molecule-1 in invasive breast cancer reflects low growth potential, negative lymph node involvement, and good prognosis. Clin. Cancer Res. 4, 31-36 (1998).

53. Anastassiou, G. et al. Expression of the cell adhesion molecules ICAM-1, VCAM1 and NCAM in uveal melanoma: a clinicopathological study. Oncology 58, 83-88 (2000).

54. Wimmenauer, S. et al. Expression of CD44, ICAM-1 and N-CAM in colorectal cancer. Correlation with the tumor stage and the phenotypical characteristics of tumor-infiltrating lymphocytes. Anticancer Res. 17, 2395-2400 (1997).

55. Yang, M., Liu, J., Piao, C., Shao, J. \& Du, J. ICAM-1 suppresses tumor metastasis by inhibiting macrophage $\mathrm{M} 2$ polarization through blockade of efferocytosis. Cell Death Dis. 6, e1780 (2015).

56. Filardy, A. A. et al. Proinflammatory clearance of apoptotic neutrophils induces an IL-12(low) IL-10(high) regulatory phenotype in macrophages. J. Immunol. 185, 2044-2050 (2010).

57. Natali, P. G. et al. Clinical significance of alpha(v)beta3 integrin and intercellular adhesion molecule-1 expression in cutaneous malignant melanoma lesions. Cancer Res. 57, 1554-1560 (1997).

58. Castets, M. et al. Inhibition of endothelial cell apoptosis by netrin-1 during angiogenesis. Dev. Cell 16, 614-620 (2009).

59. Delloye-Bourgeois, C. et al. Interference with netrin-1 and tumor cell death in non-small cell lung cancer. J. Natl. Cancer Inst. 101, 237-247 (2009).

60. Howe, A. K. Aplin, A. E. \& Juliano, R. L. Anchorage-dependent ERK signaling-mechanisms and consequences. Curr. Opin. Genet. Dev. 12, 30-35 (2002). 